\title{
RINGS AND QUASICONFORMAL MAPPINGS IN SPACE $\left({ }^{1}\right)$
}

\author{
BY \\ F. W. GEHRING( $\left.{ }^{2}\right)$
}

\section{INTRODUCTION}

This paper consists of two parts. In the first part we continue the study initiated in [7] and [9] of rings in space. We begin by establishing an inequality in $\S 3$. We next apply this inequality in $\S \S 6-7$ to show that the extremal function $u$ for a ring $R$ exists whenever both components of $\partial R$ are nondegenerate. In $\S \S 8-10$ we then show that this extremal function $u$ is real analytic provided $|\nabla u|$ is bounded away from 0 and $\infty$ a.e. on each compact set in $R$. This a priori restriction is unsatisfactory but the result is still very important since it enables us to identify the 1-quasiconformal mappings in space. In $\$ 11$ we prove a continuity property for the moduli of rings and in $\$ 12$ we use extremal lengths to obtain a pair of bounds for this modulus.

In the second part of this paper we use the results on rings developed in the first part and in [9] to study quasiconformal mappings in space. A topological mapping of a domain $D$ is said to be $K$-quasiconformal if it satisfies the two sided modulus condition

$$
K^{-1} \bmod R \leqq \bmod R^{\prime} \leqq K \bmod R
$$

for all bounded rings $R$ with $\bar{R} \subset D$. Here $R^{\prime}$ is the image of $R$ under the mapping. In $\$ 14$ we establish an important compactness property for the $K$-quasiconformal mappings of a domain. Next in $\$ \$ 15-17$ and in $\$ 20$ we study some analytic properties of quasiconformal mappings. In particular we show that they are absolutely continuous on lines and that they are differentiable with nonzero Jacobians a.e. We formulate an equivalent analytic definition for $K$-quasiconformal mappings in $\S 18$ and discuss the possibility of replacing (1) by a one sided modulus condition in $\$ 19 . \S \S 21-22$ are devoted to a third definition for quasiconformality and a theorem on exceptional sets.

In $\S \S 23-24$ we show that a quasiconformal mapping of $D$ onto $D^{\prime}$ can be extended to the boundary when $D$ and $D^{\prime}$ are spheres or half spaces. In the half space case the boundary mapping turns out to be a plane quasi-

Received by the editors July 11,1961 .

(1) The main results of this paper were first announced in May 1960 in a lecture to the Suomen Matemaattinen Yhdistys in Helsinki, Finland. They were later presented at the 1960 Summer Meeting of the American Mathematical Society in Lansing, Michigan under the titles Ring domains in space, Quasiconformal mappings in space and The Liouville theorem in space.

(2) This research was supported by Fellowships from the Guggenheim Foundation and the National Science Foundation. 
conformal mapping. We prove a general distortion theorem in $\$ 25$ which leads to an analogue of the Koebe Viertelsatz in $\$ 26$ and to an important theorem on normal families in $\$ 27$. A special distortion theorem is considered in $\$ 28$. It is the space form of a result due to Ahlfors and Mori. In $\$ 29$ we identify the 1-quasiconformal mappings and show that these are just the Moebius transformations. Then we use this result in $\$ 30$ to establish a very general form of Liouville's theorem on the conformal mappings in space which requires no a priori differentiability hypotheses. Finally in $\$ 31$ we introduce the coefficient of quasiconformality for a domain and in $\$ 32$ we give a characterization for quasiconformality based solely on the notion of compactness.

\section{RINGS IN SPACE}

1. Notation. We consider sets in finite Euclidean 3-space. Points will be designated by capital letters $P$ and $Q$ and by small letters $x$ and $y$. In the latter case $x_{1}, x_{2}$ and $x_{3}$ will denote the coordinates for $x$ and similarly for $y$. Points are treated as vectors and $|P|$ and $|x|$ will denote the norms of $P$ and $x$, respectively.

Given a point $P$ and sets $E$ and $F$ we let $\rho(P, E)$ denote the distance between $P$ and $E, \rho(E, F)$ the distance between $E$ and $F$, and $d(E)$ the diameter of $E$. We further let $\partial E, \mathfrak{e} E$ and $\bar{E}$ denote the boundary, complement and closure of $E$, respectively.

The Lebesgue 3-dimensional measure of a set $E$ will be written as $m(E)$. By the area of $E$ we mean the Hausdorff 2-dimensional measure of $E$ defined as follows:

$$
\Lambda^{2}(E)=\lim _{a \rightarrow 0+} \Lambda^{2}(E, a)=\lim _{a \rightarrow 0+}\left(\inf \sum_{U \in \mathcal{U}} \pi d(U)^{2} / 4\right) .
$$

Here, for each $a>0$, the infimum is taken over all countable coverings $u$ of $E$ by sets $U$ with $d(U) \leqq a$. A set is said to be of $\Sigma$-finite area if it is the union of sets $E_{n}$ with $\Lambda^{2}\left(E_{n}\right)<\infty$. By the length of $E$ we mean its linear or Hausdorff 1-dimensional measure

$$
\Lambda(E)=\lim _{a \rightarrow 0+} \Lambda(E, a)=\lim _{a \rightarrow 0+}\left(\inf \sum_{U \in \mathcal{U}} d(U)\right),
$$

where $\mathcal{U}$ is as above.

Finally given any function $u$ we let $\nabla u$ denote the vector $\left(\partial u / \partial x_{1}, \partial u / \partial x_{2}\right.$, $\left.\partial u / \partial x_{3}\right)$, defined at each point where these partials exist.

2. Modulus of a ring. We recall some of the definitions introduced in [9]. A ring $R$ is a domain whose complement consists of a bounded and an unbounded component which we denote as $C_{0}$ and $C_{1}$, respectively. Let $B_{0}=\partial C_{0}$ and $B_{1}=\partial C_{1}$; these are the components of $\partial R$. Then, following Loewner [16], we define the conformal capacity of $R$ as 


$$
\Gamma(R)=\inf _{u} \int_{R}|\nabla u|^{3} d \omega,
$$

where the infimum is taken over all functions $u$ which are continuously differentiable in $R$ with boundary values 0 on $B_{0}$ and 1 on $B_{1}\left({ }^{3}\right)$.

We can enlarge the class of competing functions $u$ as follows. A function $u$ is said to be absolutely continuous on lines or $A C L$ in a domain $D$ if, for each sphere $U$ with $\bar{U} \subset D, u$ is absolutely continuous on almost all line segments in $U$ parallel to the coordinate axes. If $u$ is continuous and $A C L$ in a ring $R$, then $u$ has partial derivatives a.e. in $R$. If, in addition, $u$ has boundary values 0 on $B_{0}$ and 1 on $B_{1}$, then by Lemma 1 of [9],

$$
\Gamma(R) \leqq \int_{R}|\nabla u|^{3} d \omega .
$$

We call such a function $u$ an admissible function for $R$ and we see that we can take the infimum in (2) over this class without affecting the value of $\Gamma(R)$.

Finally the modulus of a ring $R$ is defined as

$$
\bmod R=(4 \pi / \Gamma(R))^{1 / 2} .
$$

This modulus behaves in many ways like the familiar modulus of a plane ring, usually defined by means of conformal mapping. For example, if $R$ is bounded by concentric spheres of radii $a$ and $b, a<b$, then

$$
\bmod R=\log b / a \text {. }
$$

Or if $R_{1}$ and $R_{2}$ are disjoint rings, each of which separates the boundary components of $R$, then

$$
\bmod R \geqq \bmod R_{1}+\bmod R_{2} .
$$

For (5) and (6) see [9].

3. An inequality. We turn now to the problem of showing that, whenever a ring $R$ has nondegenerate boundary components, there exists an extremal admissible function $u$ for which

$$
\Gamma(R)=\int_{R}|\nabla u|^{3} d \omega .
$$

The proof is based on an argument due to Lebesgue and on the following inequality.

LEMMA 1. Let $S$ be the surface of a sphere of radius $r$ and let $u$ be a function which is continuously differentiable on $S$. Then

$$
(\underset{S}{\operatorname{osc}} u)^{3} \leqq \operatorname{Ar} \int_{S}|\nabla u|^{3} d \sigma
$$

(3) When $R$ is unbounded, this will mean that $u(x) \rightarrow 1$ as $|x| \rightarrow \infty$ in $R$. 
where

$$
A=\frac{1}{\pi}\left(\int_{0}^{\infty}\left(t+t^{8}\right)^{-1 / 2} d t\right)^{2} .
$$

The inequality (7) is best possible.

Proof. Fix $P, Q \in S$. We want to show that

$$
|u(P)-u(Q)|^{8} \leqq A r \int_{S}|\nabla u|^{8} d \sigma .
$$

Since both sides of (9) are invariant under similarity transformations, we need only consider the case where $S$ is the surface $|x|=1$ and $P$ the point $(0,0,1)$.

Now map $S$ stereographically onto $Z$, the extended complex plane $|z|=\left|x_{1}+i x_{2}\right| \leqq \infty$. Then $P$ corresponds to $z=\infty, Q$ to some point $z=a$, and hence we obtain

$$
|u(P)-u(Q)| \leqq 2 \int_{0}^{\infty}|\nabla u(z)| \frac{d t}{1+|z|^{2}}, \quad z=a+t e^{i \theta},
$$

for $0 \leqq \theta \leqq 2 \pi$. Integrating with respect to $\theta$ and applying Fubini's theorem then yields

$$
\begin{aligned}
|u(P)-u(Q)| & \leqq \frac{1}{\pi} \int_{0}^{2 \pi} \int_{0}^{\infty}|\nabla u(z)| \frac{d t d \theta}{1+|z|^{2}}, \quad z=a+t e^{i \theta} \\
& =\frac{1}{\pi} \int_{z} \frac{|\nabla u|}{|z-a|} \frac{d \sigma}{1+|z|^{2}}
\end{aligned}
$$

With Hölder's inequality we see that the last integral on the right is majorized by

$$
\frac{1}{\pi}\left(\int_{Z}|z-a|^{-3 / 2}\left(1+|z|^{2}\right)^{-1 / 2} d \sigma\right)^{2 / 3}\left(\int_{Z}|\nabla u|^{3}\left(1+|z|^{2}\right)^{-2} d \sigma\right)^{1 / 3} .
$$

It is a simple technical matter to verify that

$$
A=\sup _{|a|<\infty} \frac{1}{4 \pi^{3}}\left(\int_{z}|z-a|^{-3 / 2}\left(1+|z|^{2}\right)^{-1 / 2} d \sigma\right)^{2}<\infty
$$

and, since

$$
\int_{S}|\nabla u|^{8} d \sigma=4 \int_{Z}|\nabla u|^{8}\left(1+|z|^{2}\right)^{-2} d \sigma
$$

we obtain (9) with $r=1$ and $A$ as defined above.

In order to evaluate $A$ we assume, as we clearly may, that $a$ is real. Then 
on the basis of a theorem on the rearrangement of functions it is not difficult to verify that

$$
\begin{aligned}
\int_{-\infty}^{\infty}\left|x_{1}-a+i x_{2}\right|^{-3 / 2}\left(1+\mid x_{1}\right. & \left.+\left.i x_{2}\right|^{2}\right)^{-1 / 2} d x_{1} \\
& \leqq \int_{-\infty}^{\infty}\left|x_{1}+i x_{2}\right|^{-3 / 2}\left(1+\left|x_{1}+i x_{2}\right|^{2}\right)^{-1 / 2} d x_{1}
\end{aligned}
$$

for all $x_{2} \neq 0$. (See, for example, Theorem 378 of [13].) Integrating with respect to $x_{2}$ we thus obtain

$$
\begin{aligned}
\int_{Z}|z-a|^{-8 / 2}\left(1+|z|^{2}\right)^{-1 / 2} d \sigma & \leqq \int_{Z}|z|^{-8 / 2}\left(1+|z|^{2}\right)^{-1 / 2} d \sigma \\
& =2 \pi \int_{0}^{\infty}\left(t+t^{3}\right)^{-1 / 2} d t
\end{aligned}
$$

and hence (8) follows.

Finally for each $x \in S$ let

$$
u(x)=\int_{0}^{\phi}(\sin \theta)^{-1 / 2} d \theta
$$

where $\phi$ is the angle the radius to $x$ makes with the positive half of the $x_{8}$-axis. Then it is easy to show that (7) holds with equality, and we conclude that the inequality (7) is sharp.

We will want to appeal to the following integrated form of Lemma 1.

LEMma 2. Let $u$ be continuous and $A C L$ in $a<|x|<b$. Then

$$
\int_{a}^{b}(\underset{|x|=r}{\operatorname{osc} u})^{3} \frac{d r}{r} \leqq A \int_{a<|x|<b}|\nabla u|^{3} d \omega,
$$

where $A$ is as in Lemma 1.

Proof. We may clearly assume that $|\nabla u|$ is $L^{3}$-integrable in $a<|x|<b$ for otherwise there is nothing to prove.

Now fix $a<a^{\prime}<b^{\prime}<b$ and let

$$
v(x)=\frac{1}{m(U)} \int_{U} u(x+y) d \omega,
$$

where $U$ is the sphere $|y|<c$ and $0<c<a^{\prime}-a, b-b^{\prime}$. Then $v$ is continuously differentiable in $a^{\prime}<|x|<b^{\prime}$ and, by virtue of the $A C L$ property,

$$
|\nabla v(x)| \leqq \frac{1}{m(U)} \int_{U}|\nabla u(x+y)| d \omega
$$

there. With Minkowski's inequality and Lemma 1 it is easy to show that 


$$
\begin{aligned}
\int_{a^{\prime}}^{b^{\prime}}\left(\underset{|x|=r}{\operatorname{osc} u)^{3} \frac{d r}{r}}\right. & =\lim _{c \rightarrow 0} \int_{a^{\prime}}^{b^{\prime}}\left(\underset{|x|=r}{\operatorname{osc} v)^{3} \frac{d r}{r}}\right. \\
& \leqq \liminf _{c \rightarrow 0} A \int_{a^{\prime}<|x|<b^{\prime}}|\nabla v|^{3} d \omega \leqq A \int_{a<|x|<b}|\nabla u|^{3} d \omega,
\end{aligned}
$$

and letting $a^{\prime} \rightarrow a$ and $b^{\prime} \rightarrow b$ yields (10) as desired.

4. Extension of admissible functions. We will require the following result on the extension of admissible functions.

LEMma 3. Let $u$ be an admissible function for the ring $R$, let $|\nabla u|$ be $L^{3}$ integrable in $R$, and extend $u$ to be 0 on $C_{0}$ and 1 on $C_{1}$. Then $u$ is continuous and $A C L$ everywhere and $|\nabla u|=0$ a.e. in $\mathrm{e} R$.

Proof. The continuity is immediate. We next show that $u$ is absolutely continuous on almost all lines parallel to each of the coordinate axes. For this let $X$ denote any line parallel to the $x_{1}$-axis. Then, by Fubini's theorem,

$$
\int\left(\int_{x \cap_{R}}|\nabla u|^{3} d x_{1}\right) d x_{2} d x_{3}=\int_{R}|\nabla u|^{3} d \omega<\infty .
$$

From this and the $A C L$ property it follows that almost all lines $X$ have the following property: $u$ is absolutely continuous on each compact interval $E \subset X \cap R$ and

$$
\int_{x \cap_{R}}|\nabla u|^{3} d x_{1}<\infty
$$

Fix such a line $X$. Then using the fact that $u$ is 0 on $C_{0}$ and 1 on $C_{1}$ it is not difficult to show that

$$
|u(P)-u(Q)| \leqq \int_{E \cap_{R}}|\nabla u| d x_{1}
$$

for each compact interval $E \subset X$ with endpoints $P$ and $Q$. From this and Hölder's inequality we obtain

$$
\left(\sum_{n}\left|u\left(P_{n}\right)-u\left(Q_{n}\right)\right|\right)^{3} \leqq\left(\sum_{n}\left|P_{n}-Q_{n}\right|\right)^{2} \int_{X \cap R}|\nabla u|^{3} d x_{1},
$$

where $P_{n}$ and $Q_{n}$ are the endpoints of a sequence of nonoverlapping compact intervals in $X$. Hence $u$ is absolutely continuous on $X$ and, by symmetry, on almost all lines parallel to the coordinate axes.

Finally let $P$ be a point of $\mathfrak{C} R$ which is a point of linear density for $\mathfrak{e} R$ in the directions of the coordinate axes and at which $\nabla u$ exists. Then $\nabla u(P)$ $=0$ and, since almost all points of $e R$ satisfy the above requirements, $|\nabla u|=0$ a.e. in $\mathcal{C} R$. (See p. 298 in [27].) 
5. Monotone functions. Let $u$ be continuous in a domain $D$ and let $u$ have boundary values at each point of $\partial D$. Following Lebesgue, we say that $u$ is monotone in $D$ if

$$
\sup _{\partial \Delta} u=\sup _{\Delta} u \text { and } \inf _{\partial \Delta} u=\inf _{\Delta} u
$$

for each domain $\Delta \subset D$. Alternatively, $u$ is monotone in $D$ if and only if there exists no domain $\Delta C D$ such that $u$ is constant on $\partial \Delta$ without being constant in $\Delta$ itself. (See p. 381 in [15].)

Now let $u$ be an admissible function for a ring $R$ and let $\left\{a_{n}\right\}$ be an ordering of the rationals. We define a sequence of functions $\left\{u_{n}\right\}$ as follows. Fix $P$ in $R$. If $P$ lies in a domain $\Delta \subset R$ on whose boundary $u=a_{1}$, we set $u_{1}(P)=a_{1}$; otherwise we set $u_{1}(P)=u(P)$. We obtain $u_{2}$ from $u_{1}$ in the same way, using $a_{2}$ in place of $a_{1}$, and the sequence is defined by induction.

Next arguing as in [15], we see that the $u_{n}$ converge uniformly in $R$ to a function $v$ which is monotone in $R$ and which has the same boundary values as $u$. It is also easy to verify that

$$
\underset{B}{\operatorname{osc} v} \underset{\boldsymbol{B}}{\operatorname{osc}} u
$$

for each segment $E \subset R$. Hence $v$ is $A C L$ in $R,|\nabla v| \leqq|\nabla u|$ a.e. there, and we conclude that

$$
\Gamma(R)=\inf \int_{R}|\nabla v|^{3} d \omega
$$

where the infimum is taken over the class of admissible functions $v$ which are monotone in $R$. We call such functions monotone admissible functions.

6. Equicontinuity property. We now combine Lemmas 2 and 3 to obtain the following equicontinuity property for monotone admissible functions.

LEMMA 4. Let $R$ be a ring with nondegenerate boundary components, let $u$ be a monotone admissible function for $R$, let

$$
\int_{R}|\nabla u|^{3} d \omega \leqq M<\infty,
$$

and extend $u$ so that $u$ is 0 on $C_{0}$ and 1 on $C_{1}$. If $d\left(C_{0}\right)=b$, then

$$
|u(P)-u(Q)|^{3} \leqq A M\left(\log \frac{b}{a}\right)^{-1}
$$

whenever $|P-Q| \leqq a<b$. If $\rho\left(C_{0}, C_{1}\right)=c$, then

$$
|u(P)-u(Q)|^{3} \leqq A M\left(\log \frac{a}{c}\right)^{-1}
$$

whenever $\rho\left(P, C_{0}\right), \rho\left(Q, C_{0}\right) \geqq a>c$. Here $A$ is as in Lemma 1 . 
Proof. We begin with the proof for (11). Fix $P$ and $Q$ so that $|P-Q|$ $\leqq a<b$. Since $0 \leqq u(P), u(Q) \leqq 1$, we may assume that

$$
A M\left(\log \frac{b}{a}\right)^{-1}<1,
$$

for otherwise (11) follows trivially.

Lemma 3 implies that $u$ is continuous and $A C L$ everywhere and that $|\nabla u|=0$ a.e. in $\mathbf{e} R$. Hence we can apply Lemma 2 to conclude there exists an $r, a / 2<r<b / 2$, for which

$$
(\underset{S}{\operatorname{osc} u})^{3} \leqq A M\left(\log \frac{b}{a}\right)^{-1},
$$

where $S$ is the surface $|x-(P+Q) / 2|=r$.

Suppose first that $S \subset R$. Since the diameter of $S$ is less than that of $C_{0}$, $R$ contains $U$, the sphere bounded by $S$. Then $u$ satisfies the maximum and minimum principles in $U$ and

$$
|u(P)-u(Q)|^{3} \leqq(\underset{S}{\operatorname{osc} u})^{3} \leqq A M\left(\log \frac{b}{a}\right)^{-1}
$$

as desired.

Suppose next that $S$ meets both $R$ and $C_{0}$. Then (12) and (13) imply that $S$ does not meet $C_{1}$. Hence the boundary of each component of $U \cap R$ is contained in $C_{0} \cup S$ and, since $u$ is 0 on $C_{0}$, we conclude that

$$
|u(P)-u(Q)|^{3} \leqq\left(\sup _{S} u\right)^{3}=(\underset{S}{\operatorname{osc} u})^{3} \leqq A M\left(\log \frac{b}{a}\right)^{-1} .
$$

A slight modification of the above argument handles the case where $S$ meets $R$ and $C_{1}$.

Suppose finally that $S$ lies in a component of $\mathfrak{e} R$. Then $U$ lies in the same component, $u(P)=u(Q)$, and the proof for the first part of Lemma 4 is complete.

For the second part fix $P$ and $Q$ so that $\rho\left(P, C_{0}\right), \rho\left(Q, C_{0}\right) \geqq a>c$ and assume, as we may, that

$$
A M\left(\log \frac{a}{c}\right)^{-1}<1 .
$$

Next let $P_{0}$ be any point of $C_{0}$ for which $\rho\left(P_{0}, C_{1}\right)=c$. Then applying Lemma 2 as above we can find $r_{1}, c<r_{1}<a$, for which

$$
\left(\underset{B_{1}}{\operatorname{osc} u}\right)^{3} \leqq A M\left(\log \frac{a}{c}\right)^{-1}
$$

where $S_{1}$ is the surface $\left|x-P_{0}\right|=r_{1} . S_{1}$ clearly meets $C_{1}$ but not $C_{0}$. The 
points $P$ and $Q$ lie outside $S_{1}$ but the same argument shows there exists a second concentric spherical surface $S_{2}$ which contains these points and for which

$$
\left(\underset{S_{2}}{\operatorname{osc} u}\right)^{3} \leqq A M\left(\log \frac{a}{c}\right)^{-1}
$$

Again $S_{2}$ meets $C_{1}$ but not $C_{0}$. Hence $C_{0}$ does not meet $U$, the spherical annulus bounded by $S_{1}$ and $S_{2}$. Thus the boundary of each component of $U \cap R$ lies in $S_{1} \cup S_{2} \cup C_{1}$ and it is easy to verify that

$$
|u(P)-u(Q)|^{3} \leqq\left(\underset{S_{1} \cup S_{2}}{\operatorname{osc}} u\right)^{3} \leqq A M\left(\log \frac{a}{c}\right)^{-1}
$$

as desired.

7. Existence of the extremal function. We now combine the results of the last two sections to obtain the following existence theorem.

THEOREM 1. Let $R$ be a ring with nondegenerate boundary components. Then there exists a unique admissible function $u$ for which

$$
\Gamma(R)=\int_{R}|\nabla u|^{3} d \omega .
$$

We call $u$ the extremal function for $R$.

Proof. Let $\left\{u_{n}\right\}$ be any sequence of monotone admissible functions for $R$ such that

$$
\Gamma(R)=\lim _{n \rightarrow \infty} \int_{R}\left|\nabla u_{n}\right|^{3} d \omega .
$$

We will show that these functions converge uniformly on $R$ to the extremal function $u$.

We begin by showing that the gradients $\nabla u_{n}$ converge in the $L^{3}$-norm. For this we apply an inequality due to Clarkson (Theorem 2 of [4]) which shows the space of functions $L^{8}$-integrable over $R$ is uniformly convex. In particular we obtain

$$
\begin{aligned}
\int_{R}\left|\frac{\nabla u_{m}-\nabla u_{n}}{2}\right|^{8} d \omega+\int_{R}\left|\frac{\nabla u_{m}+\nabla u_{n}}{2}\right|^{3} d \omega & \\
& \leqq \frac{1}{2} \int_{R}\left|\nabla u_{m}\right|^{3} d \omega+\frac{1}{2} \int_{R}\left|\nabla u_{n}\right|^{8} d \omega
\end{aligned}
$$

for all $m$ and $n$. Then, since $\left(u_{m}+u_{n}\right) / 2$ is admissible for $R$, this inequality yields 
$\limsup _{m, n \rightarrow \infty} \int_{R}\left|\frac{\nabla u_{m}-\nabla u_{n}}{2}\right|^{3} d \omega=\Gamma(R)-\liminf _{m, n \rightarrow \infty} \int_{R}\left|\frac{\nabla u_{m}+\nabla u_{n}}{2}\right|^{3} d \omega=0$.

Hence the $\nabla u_{n}$ converge in the $L^{3}$-norm to a vector function $f=\left(f_{1}, f_{2}, f_{8}\right)$ and

$$
\Gamma(R)=\int_{R}|f|^{3} d \omega .
$$

Next let $b=d\left(C_{0}\right)$ and $c=\rho\left(C_{0}, C_{1}\right)$, let $M$ be a constant such that

$$
\int_{R}\left|\nabla u_{n}\right|^{3} d \omega \leqq M<\infty
$$

for all $n$, and extend $u_{n}$ to be 0 on $C_{0}$ and 1 on $C_{1}$. We see from Lemma 4 that

$$
\left|u_{n}(P)-u_{n}(Q)\right|^{3} \leqq A M\left(\log \frac{b}{a}\right)^{-1}
$$

for all $n$ whenever $|P-Q| \leqq a<b$ and that

$$
\left|u_{n}(P)-u_{n}(Q)\right|^{3} \leqq A M\left(\log \frac{a}{c}\right)^{-1}
$$

for all $n$ whenever $\rho\left(P, C_{0}\right), \rho\left(Q, C_{0}\right) \geqq a>c$. Ascoli's theorem then implies that a subsequence $\left\{u_{n_{k}}\right\}$ converges uniformly for all $x$ to a function $u$.

We show that $u$ is admissible for $R$ and that $\nabla u=f$ a.e. First it is clear that $u$ is continuous in $R$ and has boundary values 0 on $B_{0}$ and 1 on $B_{1}$. Next appealing to Fubini's theorem, Hölder's inequality and the $A C L$ property of the $u_{n}$, it is not difficult to verify that almost all lines $X$, parallel to the $x_{1}$-axis, have the following property: if $E$ is a compact interval in $X \cap R$ with endpoints $P$ and $Q$, then

$$
\int_{E} f_{1} d x_{1}=\lim _{k \rightarrow \infty} \int_{E} \frac{\partial u_{n_{k}}}{\partial x_{1}} d x_{1}=\lim _{k \rightarrow \infty}\left(u_{n_{k}}(P)-u_{n_{k}}(Q)\right)=u(P)-u(Q) .
$$

From this it follows that $u$ is absolutely continuous on $X \cap R$ with $\partial u / \partial x_{1}=f_{1}$ a.e. in this linear set. Thus, by symmetry, $u$ is $A C L$ in $R, \nabla u=f$ a.e. there and hence $u$ has the desired extremal property.

To complete the proof of the theorem we show that $u$ is unique. In particular we prove that if $u$ and $v$ are admissible functions for $R$ and if

$$
\Gamma(R)=\int_{R}|\nabla u|^{3} d \omega=\int_{R}|\nabla v|^{3} d \omega
$$

then $u=v$ in $R$.

For this let $w=u-v$ and consider for real $t$ the integral 


$$
W(t)=\int_{R}|\nabla u+t \nabla w|^{3} d \omega
$$

Since $u+t w$ is admissible for $R, W(0) \leqq W(t)$ for all $t$. Hölder's inequality and the Lebesgue dominated convergence theorem allow us to differentiate $W(t)$ with respect to $t$ under the integral sign. Then setting $t=0$ yields

$$
\int_{R}|\nabla u| \nabla u \cdot \nabla w d \omega=0
$$

where $\nabla u \cdot \nabla w$ is the scalar product of $\nabla u$ and $\nabla w$. Thus

$$
\Gamma(R)=\int_{R}|\nabla u| \nabla u \cdot \nabla v d \omega \leqq \int_{R}|\nabla u|^{2}|\nabla v| d \omega
$$

while Hölder's inequality yields

$$
\int_{R}|\nabla u|^{2}|\nabla v| d \omega \leqq\left(\int_{R}|\nabla u|^{3} d \omega\right)^{2 / 3}\left(\int_{R}|\nabla v|^{3} d \omega\right)^{1 / 3}=\Gamma(R) .
$$

Hence we have equality throughout in both (15) and (16). Equality in (16) implies that $|\nabla u|$ and $|\nabla v|$ are proportional a.e. in $R$ and hence that $|\nabla u|=|\nabla v|$ a.e. From the equality in (15) we conclude that $\nabla u \cdot \nabla v=|\nabla u||\nabla v|$ a.e. in $R$. Thus $\nabla u=\nabla v$ a.e. and, because $u$ and $v$ are both admissible functions, it follows that $u=v$ everywhere in $R$.

The fact that $u$ is unique allows us to conclude that the original sequence $\left\{u_{n}\right\}$ converges uniformly to $u$ on $R$. For if this were not the case, we could find a second subsequence which would converge to a function $v$ different from $u$. But $v$ would have the same extremal property as $u$, thus contradicting the uniqueness of $u$.

Hence we have established the following result.

COROLLARY 1. If $R$ has nondegenerate boundary components and if $\left\{u_{n}\right\}$ is a sequence of monotone admissible functions for which

$$
\Gamma(R)=\lim _{n \rightarrow \infty} \int_{R}\left|\nabla u_{n}\right|^{3} d \omega
$$

then the $u_{n}$ converge to the extremal function $u$ uniformly in $R$.

We also list here the variational condition satisfied by the extremal function.

COROLlaRy 2. Let $u$ be the extremal function for $R$ and let $w$ be a function which is continuous and $A C L$ in $R$, which has boundary value 0 on $\partial R$ and for which $|\nabla w|$ is $L^{3}$-integrable in $R$. Then 


$$
\int_{R}|\nabla u| \nabla u \cdot \nabla w d \omega=0 .
$$

Finally we observe that the extremal function $u$ is monotone in $R$. For suppose there exists a domain $\Delta C R$, such that $u$ is equal to a constant $a$ on $\partial \Delta$ without being constant in $\Delta$, and set $v$ equal to $u$ in $R-\Delta$ and to $a$ in $\Delta$. Then $v$ is admissible for $R$ and, arguing as in the last part of Lemma 3, we see that $|\nabla u|=|\nabla v|$ a.e. in $R-\Delta$. Hence

$$
\Gamma(R) \leqq \int_{R}|\nabla v|^{3} d \omega=\int_{R}|\nabla u|^{3} d \omega-\int_{\Delta}|\nabla u|^{8} d \omega<\int_{R}|\nabla u|^{8} d \omega
$$

and this contradicts the extremal property of $u$.

8. Analyticity of the extremal function. The extremal function for a space ring $R$ plays a role similar to that of the harmonic measure of a plane ring whose Dirichlet integral yields the electrostatic capacity of the plane ring. Since this harmonic measure is always real analytic, one would expect that the same is true of the extremal function for a space ring.

The following result is a step in this direction which enables us to identify the 1-quasiconformal mappings in space and to establish a very general form of Liouville's theorem.

THEOREM 2. Let $u$ be the extremal function for a ring $R$ and, for each compact set $E \subset R$, let a positive constant $M$ exist such that

$$
\frac{1}{M} \leqq|\nabla u| \leqq M
$$

a.e. in $E$. Then $u$ is real analytic and satisfies the differential equation

$$
\operatorname{div}(|\nabla u| \nabla u)=0
$$

everywhere in $R$.

The proof is divided into several steps. First we use an important recent result due to de Giorgi to show that $u$ is continuously differentiable. Next a theorem of Morrey's allows us to pass from $C^{1}$ to $C^{n}$. Then we can apply the Gauss theorem to Corollary 2 to obtain (19). The analyticity finally follows from a theorem due to $E$. Hopf.

9. Proof for continuous differentiability. Let $D$ denote any bounded domain whose closure lies in $R$, let $b=\rho(D, \partial R)$ and fix $0<a<b$. The set of points $x$ for which $\rho(x, D) \leqq a$ is a compact subset of $R$ and hence we can find a positive $M$ such that (18) holds a.e. in this set.

Now let $h$ denote a vector parallel to the positive half of the $x_{1}$-axis with $0<|h| \leqq a$. Then

$$
\frac{1}{M} \leqq|\nabla u(x)|,|\nabla u(x+h)| \leqq M
$$


a.e. in $D$. Next let

$$
v(x)=\frac{u(x+h)-u(x)}{|h|} .
$$

Then $v$ is continuous and $A C L$ in $D$ and from (20) it is easy to verify that $|v| \leqq M$ everywhere in $D$.

We will use the following result due to de Giorgi [11] to show that the functions $v$ are equicontinuous in $D$ as $|h| \rightarrow 0$. (For this particular form of de Giorgi's theorem see [21].)

Lemma 5. Let the functions $a_{i j}$ be measurable in $D$ for $i, j=1,2,3$, let $a_{i j}=a_{j i}$ and let positive constants $M_{1}$ and $M_{2}$ exist such that for each vector $f=\left(f_{1}, f_{2}, f_{3}\right)$

$$
M_{1}|f|^{2} \leqq \sum_{i, j} a_{i j} f_{i} f_{j} \leqq M_{2} \mid f !^{2}
$$

a.e. in D. Next let $v$ be continuous and $A C L$ in $D$, let

$$
\int_{D}^{2} d \omega \leqq M_{3}<\infty,
$$

let $|\nabla v|$ be $L^{2}$-integrable in $D$ and let

$$
\int_{D} \sum_{i, j} a_{i j} \frac{\partial v}{\partial x_{i}} \frac{\partial w}{\partial x_{j}} d w=0
$$

for all continuously differentiable functions w with compact support in $D$. Then, given any compact set $E \subset D$, there exist constants $A$ and $\alpha$, which depend only on the $M_{i}$ and $E$, such that

$$
|\vartheta(P)-v(Q)| \leqq A|P-Q|^{\alpha}
$$

for all $P$ and $Q$ in $E$.

We now use the variational condition (17) to show that the function $v$ defined in (21) satisfies the hypotheses of Lemma 5.

First let $w$ be continuously differentiable with compact support in $D$. Then $w(x)$ and $w(x-h)$ vanish on $\partial R$ and Corollary 2 yields

$$
\int_{D}(|\nabla u(x+h)| \nabla u(x+h)-|\nabla u(x)| \nabla u(x)) \cdot \nabla w(x) d \omega=0 .
$$

Now let

$$
\begin{aligned}
g & =\frac{1}{3}|y|^{3}, \quad g_{j}=\frac{\partial g}{\partial y_{j}}=|y| y_{j}, \\
g_{i j} & =\frac{\partial^{2} g}{\partial y_{i} \partial y_{j}}=|y| \delta_{i j}+\frac{1}{|y|} y_{i} y_{j}
\end{aligned}
$$


where $\delta_{i j}$ is the Kronecker delta, and let

$$
y=t \nabla u(x+h)+(1-t) \nabla u(x),
$$

Then we obtain

$$
\int_{D} \sum_{j}\left(g_{j}(y(1))-g_{j}(y(0))\right) \frac{\partial w}{\partial x_{j}} d \omega=0 .
$$

It is easy to verify that

$$
g_{j}(y(1))-g_{j}(y(0))=|h| \sum_{i}\left(\int_{0}^{1} g_{i j}(y) d t\right) \frac{\partial v}{\partial x_{i}}
$$

and we conclude that (24) holds with

$$
a_{i j}=\int_{0}^{1} g_{i j}(y) d t .
$$

The $a_{i j}$ are clearly measurable in $D$ and $a_{i j}=a_{j i}$. Next for each vector $f$ we have

$$
\sum_{i, j} a_{i j} f_{i} f_{j}=\int_{0}^{1}\left(|y||f|^{2}+\frac{(y \cdot f)^{2}}{|y|}\right) d t
$$

and thus

$$
|f|^{2} \int_{0}^{1}|y| d t \leqq \sum_{i, j} a_{i j} f_{i} f_{j} \leqq 2|f|^{2} \int_{0}^{1}|y| d t .
$$

If we pick an $x$ where (20) holds, then

$$
\frac{1}{4 M} \leqq \int_{0}^{1}|y| d t \leqq M
$$

and hence (22) holds a.e. in $D$ with $M_{1}=1 / 4 M$ and $M_{2}=2 M$. Finally, since $|v| \leqq M$ in $D$, (23) holds and we can apply Lemma 5 to conclude that the functions $v$ satisfy a Hölder condition at each point of $D$, uniformly in $h$.

Now by Ascoli's theorem there exists a sequence $\left\{h_{n}\right\},\left|h_{n}\right| \rightarrow 0$, such that

$$
\lim _{n \rightarrow \infty} \frac{u\left(x+h_{n}\right)-u(x)}{\left|h_{n}\right|}
$$

exists uniformly on each compact subset of $D$. It then follows that $\partial u / \partial x_{1}$ exists and is continuous everywhere in $D$; by symmetry $u$ is thus continuously differentiable in $D$.

10. Completion of proof for Theorem 2. Again let $w$ be any continuously differentiable function with compact support in $D$. Then Corollary 2 yields 


$$
\int_{D} \nabla g(\nabla u) \cdot \nabla w d \omega=0
$$

where $g$ is as in (25). Next (20) and the continuity of $\nabla u$ imply that, for each vector $f$,

$$
\frac{1}{M}|f|^{2} \leqq \sum_{i, j} g_{i j}(\nabla u) f_{i} f_{j} \leqq 2 M|f|^{2}
$$

everywhere in $D$. Since $g(y)$ is analytic in $|y|>0$ we can apply a theorem of Morrey's (Theorem 9.2 of [20]) to conclude that $u$ has continuous derivatives of all orders.

Now let $U$ be any sphere with $\bar{U} \subset D$. Then the Gauss theorem yields

$$
\int_{U} \operatorname{div}(w|\nabla u| \nabla u) d \omega=\int_{\partial U} w|\nabla u| \frac{\partial u}{\partial n} d \sigma,
$$

( $n$ outer normal to $\partial U$ ), for each continuously differentiable function $w$. If $w$ has, in addition, compact support in $U$, then Corollary 2 and the above yield

$$
\int_{U} w \operatorname{div}(|\nabla u| \nabla u) d \omega=0 .
$$

From this it follows that

$$
\operatorname{div}(|\nabla u| \nabla u)=0
$$

in $U$ and hence everywhere in $D$. In particular $u$ is a solution of the quasilinear equation

in $D$.

$$
\sum_{i, j}\left(\delta_{i j}|\nabla u|^{2}+\frac{\partial u}{\partial x_{i}} \frac{\partial u}{\partial x_{j}}\right) \frac{\partial^{2} u}{\partial x_{i} \partial x_{j}}=0
$$

The above equation is elliptic in $D$ and, since the coefficients of $\partial^{2} u / \partial x_{i} \partial x_{j}$ are analytic functions of the partial derivatives $\partial u / \partial x_{i}$, we can appeal to a theorem due to E. Hopf [14] to conclude that $u$ is real analytic in $D$. Now $D$ was chosen to be any bounded domain with closure in $R$. Thus the above results hold throughout $R$ and the proof for Theorem 2 is complete.

11. Continuity of the modulus. A sequence of sets $\left\{E_{n}\right\}$ is said to converge uniformly to a set $E$ if, for each $\epsilon>0$, there exists an $N$ such that $n>N$ implies each point of $E_{n}$ lies within distance $\epsilon$ of $E$ and each point of $E$ lies within distance $\epsilon$ of $E_{n}$.

We establish next the following continuity property for the modulus of a ring. (See also [8].)

LEMMA 6. Let $\left\{R_{n}\right\}$ be a sequence of rings and let $R$ be a bounded ring. If each of the components of $\partial R_{n}$ converges uniformly to the corresponding component of $\partial R$, then 


$$
\bmod R=\lim _{n \rightarrow \infty} \bmod R_{n}
$$

Proof. Suppose first that $C_{0}$ reduces to a point $P$ and fix $0<a<b<\rho\left(P, C_{1}\right)$. Then $a<|x-P|<b$ separates the boundary components of $R_{n}$ for large $n$. Hence

$$
\liminf _{n \rightarrow \infty} \bmod R_{n} \geqq \log \frac{b}{a}
$$

and, since mod $R=\infty$, holding $b$ fixed and letting $a \rightarrow 0$ yields (26) as desired.

Now suppose that $C_{0}$ is nondegenerate, let $u$ be the extremal function for $R$ and extend $u$ so that $u$ is 0 on $C_{0}$ and 1 on $C_{1}$. Next fix $0<a<1 / 2$ and pick $b>0$ so that $|u(P)-u(Q)| \leqq a$ whenever $|P-Q| \leqq b$. If we define

$$
v=\left\{\begin{array}{cl}
0 & \text { if } u<a, \\
\frac{u-a}{1-2 a} & \text { if } a \leqq u \leqq 1-a, \\
1 & \text { if } 1-a<u,
\end{array}\right.
$$

then $v$ is continuous and $A C L$ everywhere. Now $v$ is 0 and 1 at points within distance $b$ of $C_{0}$ and $C_{1}$, respectively, and hence $v$ is admissible for $R_{n}$ when $\boldsymbol{n}$ is large. Thus

$$
\Gamma\left(R_{n}\right) \leqq \int_{R_{n}}|\nabla v|^{8} d \omega \leqq(1-2 a)^{-8} \int_{R}|\nabla u|^{8} d \omega=(1-2 a)^{-8} \Gamma(R)
$$

for $n>N(a)$, and letting $a \rightarrow 0$ yields

$$
\limsup _{n \rightarrow \infty} \Gamma\left(R_{n}\right) \leqq \Gamma(R) .
$$

To complete the proof for (26) we show that

$$
\lim _{n \rightarrow \infty} \inf \Gamma\left(R_{n}\right) \geqq \Gamma(R) \text {. }
$$

Since $B_{n, 0}$ converges uniformly to $B_{0}$, we may assume that $d\left(C_{n, 0}\right) \geqq c>0$ for all $n$. Next let $u_{n}$ be the extremal function for $R_{n}$ and extend $u_{n}$ to be 0 on $C_{n, 0}$ and 1 on $C_{n, 1}$. Inequality (28) implies we can find an $M<\infty$ such that

$$
\Gamma\left(R_{n}\right)=\int_{R_{n}}\left|\nabla u_{n}\right|^{8} d \omega \leqq M
$$

for all $n$. Fix $0<a<1 / 2$. Then Lemma 4 implies that $\left|u_{n}(P)-u_{n}(Q)\right| \leqq a$ whenever $|P-Q| \leqq b$, where

$$
b=c \exp \left(-A M / a^{3}\right) .
$$

If we define $v_{n}$ as in (27), with $u_{n}$ in place of $u$, then $v_{n}$ will be admissible for $R$ when $n$ is large. Thus 


$$
\Gamma(R) \leqq \int_{R}\left|\nabla v_{n}\right|^{3} d \omega \leqq(1-2 a)^{-3} \int_{R n}\left|\nabla u_{n}\right|^{3} d w=(1-2 a)^{-3} \Gamma\left(R_{n}\right)
$$

for $n>N(a)$, and letting $a \rightarrow 0$ yields (29) completing the proof for Lemma 6 .

12. Extremal length estimates. We conclude the first part of this paper by deriving a pair of rough bounds for the conformal capacity of a ring using extremal lengths.

Given a ring $R$ we let $l(R)=\rho\left(C_{0}, C_{1}\right)$ and

$$
s(R)=\inf _{E} \Lambda^{2}(E),
$$

where $E$ is any set in $R$ which separates $C_{0}$ and $C_{1}$. We then have the following space form of Rengel's inequality for rings.

Lemma 7. Let $R$ be a ring. Then

$$
\frac{s(R)^{3}}{m(R)^{2}} \leqq \Gamma(R) \leqq \frac{m(R)}{l(R)^{3}} .
$$

Proof. Let $u$ be a continuously differentiable admissible function for $R$ and, for each $0<a<1$, let $E$ denote the set where $u=a$. Since $E$ lies in $R$ and separates $C_{0}$ and $C_{1}$,

$$
s(R) \leqq \Lambda^{2}(E)
$$

for $0<a<1$. Then $u$ satisfies a uniform Lipschitz condition on each compact subset of $R$ and a recent result due to Federer and Young (see p. 426 of [5]) gives

$$
s(R) \leqq \int_{0}^{1} \Lambda^{2}(E) d a \leqq \int_{R}|\nabla u| d \omega .
$$

Hölder's inequality implies that

$$
s(R)^{3} \leqq m(R)^{2} \int_{R}|\nabla u|^{3} d \omega
$$

and taking the infimum over all such $u$ yields the first inequality.

For the second inequality let

$$
u(x)=\min .\left(1, \frac{\rho\left(x, C_{0}\right)}{l(R)}\right) .
$$

Then $u$ is admissible for $R$ and $|\nabla u| \leqq 1 / l(R)$ a.e. in $R$. Hence

$$
\Gamma(R) \leqq \int_{R}|\nabla u|^{3} d \omega \leqq \frac{m(R)}{l(R)^{3}}
$$

and the proof is complete. 


\section{QUASICONFORMAL MAPPINGS IN SPACE}

13. Definitions. We now use the results on rings proved in the first part of this paper and in [9] to discuss quasiconformal mappings in space. We begin with some notation.

Let $y(x)$ be a topological mapping (homeomorphism) of a domain $D$. We let $E^{\prime}$ denote the image of each set $E \subset D$ under $y(x)$; in particular $D^{\prime}$ will always denote the image of $D$. Conversely $E$ will denote the image of each set $E^{\prime} \subset D^{\prime}$ under the inverse mapping $x(y)$. Similarly we let $P^{\prime}=y(P)$ for all points $P$ in $D$ and $P=x\left(P^{\prime}\right)$ for all points $P^{\prime}$ in $D^{\prime}$.

Next we introduce the following functions to measure various distortions of $y(x)$ at a point $P$ in $D$. First we let

$$
H(P)=\limsup _{r \rightarrow 0} \frac{L(P, r)}{l(P, r)}, \quad I(P)=\limsup _{r \rightarrow 0} \frac{L(P, r)}{r},
$$

where for $0<r<\rho(P, \partial D)$,

$$
L(P, r)=\max _{|P-Q|=r}\left|P^{\prime}-Q^{\prime}\right|, \quad l(P, r)=\min _{|P-Q|=r}\left|P^{\prime}-Q^{\prime}\right| .
$$

Next we let

$$
J(P)=\underset{r \rightarrow 0}{\limsup } \frac{m\left(U^{\prime}\right)}{m(U)},
$$

where $U$ is the open sphere with center at $P$ and radius $r$. At a point of differentiability $x, I(x)$ is the maximum stretching, $H(x)$ is the ratio of the maximum to minimum stretching and $J(x)$ is the absolute value of the Jacobian. Finally we let $H^{*}(y), I^{*}(y), L^{*}(y, r), l^{*}(y, r)$ and $J^{*}(y)$ denote the corresponding functions for the inverse mapping $x(y)$.

We adopt the following definition for quasiconformality.

Definition. A topological mapping $y(x)$ of a domain $D$ is said to be $K$ quasiconformal, $1 \leqq K<\infty$, if

$$
\frac{1}{K} \bmod R \leqq \bmod R^{\prime} \leqq K \bmod R
$$

for all bounded rings $R$ with $\bar{R} \subset D$. A quasiconformal mapping is one which is $K$-quasiconformal for some $K\left({ }^{4}\right)$.

Let $y(x)$ be a topological mapping of $D$, let $y(x)$ be continuously differentiable with nonvanishing Jacobian and let $y(x)$ map infinitesimal spheres onto infinitesimal ellipsoids so that the ratio of the major to minor axes does

(4) J. Väisälä defines a class of $K$-quasiconformal mappings in [28] which coincides with the class of $K^{1 / 2}$-quasiconformal mappings by the above definition. He uses extremal lengths to study these mappings and has obtained independently analogues for Theorems 4, 5, 6, 9 and 10. 
not exceed $K$, that is $H(x) \leqq K$ everywhere in $D$. Then it is easy to see that $y(x)$ satisfies (30) for all rings $R$ with $\bar{R} \subset D$, and hence $y(x)$ is $K$-quasiconformal by the above definition.

A Moebius transformation is an element of the group of transformations of the extended space onto itself generated by similarity mappings and inversions. If $y(x)$ is a topological mapping which is the restriction of a Moebius transformation to a domain $D$, then it follows that $y(x)$ is a 1-quasiconformal mapping. We will show in $\$ 29$ that the converse is true, that is every 1 -quasiconformal mapping of $D$ is the restriction of a Moebius transformation to $D$.

Finally it is clear from (30) that the inverse of a $K$-quasiconformal mapping is itself $K$-quasiconformal and that the composition of two mappings which are $K_{1}$ and $K_{2}$-quasiconformal is itself a $K_{1} K_{2}$-quasiconformal mapping.

14. Compactness property. The following compactness property illustrates one of the advantages of the above definition for quasiconformality over the classical one.

THEOREM 3. Let $\left\{y_{n}(x)\right\}$ be a sequence of $K$-quasiconformal mappings of a domain $D$ which converge uniformly on each compact subset of $D$ to a topological mapping $y(x)$. Then $y(x)$ is $K$-quasiconformal.

Proof. Let $R$ be a bounded ring with $\bar{R} \subset D$, and let $R_{n}^{\prime}$ and $R^{\prime}$ denote the images of $R$ under $y_{n}(x)$ and $y(x)$, respectively. Then $R^{\prime}$ is a bounded ring and each of the components of $\partial R_{n}^{\prime}$ converges uniformly to the corresponding component of $\partial R^{\prime}$. Hence, by Lemma 6,

$$
\bmod R^{\prime}=\lim _{n \rightarrow \infty} \bmod R_{n}^{\prime}
$$

and, since

$$
\frac{1}{K} \bmod R \leqq \bmod R_{n}^{\prime} \leqq K \bmod R
$$

for all $n$, we conclude that $y(x)$ is itself a $K$-quasiconformal mapping.

15. Upper bound for $H(x)$. We want next to derive some of the analytic properties of quasiconformal mappings. We begin with the following space form of a result due to Mori [19].

LEMMA 8. Let $y(x)$ be a topological mapping of a domain $D$ and let

$$
\bmod R^{\prime} \leqq K \bmod R
$$

for all bounded rings $R$ with $\bar{R} \subset D$. Then $H(x)<e^{6 K}$ everywhere in $D$.

Proof. Fix $P$ in $D$ and fix $a>0$ so that $L(P, a)<\rho\left(P^{\prime}, \partial D^{\prime}\right)$. Next for $0<r<a$ let $R^{\prime}$ be the spherical annulus $l(P, r)<\left|y-P^{\prime}\right|<L(P, r)$ and let $R$ be the preimage of $R^{\prime}$ under $y(x)$. Then $P$ is in $C_{0}$ and both $C_{0}$ and $C_{1}$ contain 
points of $|x-P|=r$. Hence Theorem 4 of [9] yields the upper bound $\bmod R \leqq \log \Psi(1)<6$,

and with (31) we obtain

$$
\log \frac{L(P, r)}{l(P, r)}=\bmod R^{\prime} \leqq K \bmod R \leqq K \log \Psi(1)<6 K
$$

for $0<r<a$. The desired conclusion now follows directly.

16. Absolute continuity on lines. From Lemma 8 we see that $\dot{H}(x)$ is bounded whenever $y(x)$ is quasiconformal. The next result allows us to conclude from this fact that the mapping and its inverse are $A C L$.

LeMma 9. Let $y(x)$ be a topological mapping of a domain $D$ and let $H(x)$ be bounded everywhere in $D$. Then $y(x)$ is $A C L$ in $D$ and $x(y)$ is $A C L$ in $D^{\prime}$.

Proof. We consider only the argument for $x(y)$ in detail. Given any sphere $U^{\prime}$ with $\bar{U}^{\prime} \subset D^{\prime}$, we want to show that $x(y)$ is absolutely continuous on almost all segments in $U^{\prime}$ parallel to each of the coordinate axes.

Let $Y^{\prime}$ be any line parallel to the $y_{1}$-axis. Next for $r>0$ let $G^{\prime}=G^{\prime}(r)$ denote the set of points in $U^{\prime}$ which lie within a distance $r$ of $Y^{\prime}$. Then, because $U$ is bounded, we can apply Lebesgue's theorem (see p. 115 of [27]) to conclude that

$$
T\left(Y^{\prime}\right)=\limsup _{r \rightarrow 0} \frac{m(G)}{r^{2}}<\infty
$$

for almost all lines $Y^{\prime}$. Let $Y^{\prime}$ be any such line and let $E^{\prime}$ be a compact set in $U^{\prime} \cap Y^{\prime}$. We shall show that

$$
\Lambda(E)^{3} \leqq A T\left(Y^{\prime}\right) \Lambda\left(E^{\prime}\right)^{2},
$$

where $A$ is a constant which depends only on the bound for $H(x)$.

Fix $a$ so that $H(x)<a$ in $E$ and, for $0<b<\rho(E, \partial U)$, let $E_{b}$ be the set of $P$ in $E$ for which

$$
L(P, s) \leqq a l(P, s)
$$

whenever $0<s \leqq b$. Since $E_{b}$ is compact and expands to $E$ as $b \rightarrow 0$, a simple limiting argument shows it is sufficient to establish (32) with $E_{b}$ in place of $E$. In other words we may assume that (33) holds for all $P$ in $E$.

Pick $c>0$ so that $L^{*}\left(P^{\prime}, c\right) \leqq b$ for $P^{\prime}$ in $E^{\prime}$ and fix $u<d<c$. By Lemma 1 of [6] we can find $0<t<d$ and points $P_{1}^{\prime}, \cdots, P_{N}^{\prime}$ in the linear set $E^{\prime}$ such that the spheres $\left|y-P_{n}^{\prime}\right|<t$ cover $E^{\prime}$,

$$
\left|P_{i}^{\prime}-P_{j}^{\prime}\right| \geqq|i-j| t
$$

for $i, j=1, \cdots, N$ and 


$$
N t \leqq \Lambda\left(E^{\prime}\right)+d .
$$

Next let $U_{n}$ be the sphere $\left|x-P_{n}\right|<s_{n}$ where $s_{n}=L^{*}\left(P_{n}^{\prime}, t\right)$. These spheres cover $E$ and $0<s_{n} \leqq b$. Hence

$$
\Lambda(E, 2 b) \leqq \sum_{n} 2 s_{n}
$$

and Hölder's inequality yields

$$
\Lambda(E, 2 b)^{3} \leqq \frac{6}{\pi} N^{2} \sum_{n} m\left(U_{n}\right) .
$$

From (33) we see that

$$
L\left(P_{n}, s_{n}\right) \leqq a l\left(P_{n}, s_{n}\right)=a t .
$$

Thus each point of $U_{n}^{\prime}$ lies within distance at of $P_{n}^{\prime}$ and hence in the set $G^{\prime}=G^{\prime}(a t)$. Moreover (34) and (35) imply that each point of $G^{\prime}$ lies in at most $[3 a]$ different $U_{n}^{\prime}$. Hence

$$
\Lambda(E, 2 b)^{3} \leqq \frac{18 a^{3}}{\pi} \frac{m(G)}{(a t)^{2}}(N t)^{2} \leqq A \frac{m(G)}{r^{2}}\left(\Lambda\left(E^{\prime}\right)+d\right)^{2}
$$

where $0<r=a t<a d$. Letting $d \rightarrow 0$ yields

$$
\Lambda(E, 2 b)^{3} \leqq A T\left(Y^{\prime}\right) \Lambda\left(E^{\prime}\right)^{2}
$$

and, since this holds for all small $b>0$, we obtain (32) as desired.

Finally from (32) it follows that $x(y)$ is absolutely continuous on $U^{\prime} \cap Y^{\prime}$ for almost all lines $Y^{\prime}$ parallel to the $y_{1}$-axis. Hence, by symmetry, $x(y)$ is $A C L$ in $D^{\prime}$.

The proof that $y(x)$ is $A C L$ in $D$ is very similar. Let $U$ be a sphere with $\bar{U} \subset D$. Then for almost all lines $X$ parallel to the $x_{1}$-axis

$$
T(X)=\underset{r \rightarrow 0}{\lim \sup } \frac{m\left(G^{\prime}\right)}{r^{2}}<\infty,
$$

where $G$ is the set of points in $U$ within distance $r$ of $X$. When $T(X)<\infty$, a simplified version of the above argument shows that

$$
\Lambda\left(E^{\prime}\right)^{3} \leqq A T(X) \Lambda(E)^{2}
$$

for each compact set $E \subset U \cap X$, where $A$ depends only on the bound for $H(x)$. (See also the proof for Theorem 2 in [6].) The $A C L$ property for $y(x)$ then follows as above and the proof for Lemma 9 is complete.

17. Differentiability. We consider first some relations between the distortion functions for a topological mapping and its inverse.

Lemma 10. Let $y(x)$ be a topological mapping of a domain $D$ and let $P$ be $a$ point of $D$. If $H(P)<\infty$, then 


$$
I(P)^{3} \leqq H(P)^{3} J(P), \quad I^{*}\left(P^{\prime}\right)^{3} \leqq H(P)^{3} J^{*}\left(P^{\prime}\right) .
$$

If, in addition, $y(x)$ is differentiable at $P$, then

$$
I(P)^{3} \leqq H(P)^{2} J(P) .
$$

Proof. We verify only the second part of (36). For this let $s=L^{*}\left(P^{\prime}, r\right)$ and $t=L(P, s)$ for all small $r>0$. Then $r=l(P, s), s=l^{*}\left(P^{\prime}, t\right)$ and we have

$$
\begin{aligned}
I^{*}\left(P^{\prime}\right)^{3} & =\left(\limsup _{r \rightarrow 0} \frac{s}{r}\right)^{3} \\
& \leqq\left(\limsup _{r \rightarrow 0} \frac{t}{r}\right)^{3}\left(\limsup _{r \rightarrow 0} \frac{s}{t}\right)^{3} \leqq H(P)^{3} J^{*}\left(P^{\prime}\right)
\end{aligned}
$$

as desired.

Now let $y(x)$ be a topological mapping of a domain $D$ and let

$$
\bmod R^{\prime} \leqq K \bmod R
$$

for all bounded rings $R$ with $\bar{R} \subset D$. Then $H(x)$ is bounded in $D$ and $y(x)$ and $x(y)$ are $A C L$ by Lemma 9 . Next for each Borel set $E \subset D$ let $\tau(E)=m\left(E^{\prime}\right)$. Then $\tau$ is an additive set function and, by Lebesgue's theorem, its upper symmetric derivate $J(x)$ is finite a.e. in $D$. Arguing similarly for $x(y)$ and then applying (36) we conclude that $I(x)$ and $I^{*}(y)$ are finite a.e. in $D$ and $D^{\prime}$, respectively. The Rademacher-Stepanoff theorem then implies that $y(x)$ and $x(y)$ are a.e. differentiable. (See p. 201 of [24].)

We examine what happens in the neighborhood of these points of differentiability.

LemMA 11. Let $y(x)$ be a topological mapping of a domain $D$ and let (37) hold for all bounded rings $R$ with $\bar{R} \subset D$. Then

$$
I(x)^{3} \leqq K^{2} J(x)
$$

wherever $y(x)$ is differentiable and

$$
I^{*}(y)^{3} \leqq K^{4} J^{*}(y)
$$

wherever $x(y)$ is differentiable.

Proof. We begin by establishing (38) at each point $P$ in $D$ where $y(x)$ is differentiable. If $J(P)=0$, then (38) follows from (36) of Lemma 10 and hence we may assume that $J(P)>0$. Next by performing preliminary translations, rotations and reflections we can reduce our problem to the case where $P=$ $P^{\prime}=0$ and $y(x)$ is of the form

$$
y_{i}=a_{i} x_{i}+o(|x|)
$$

$$
i=1,2,3 \text {, }
$$

with $0<a_{1} \leqq a_{2} \leqq a_{3}$. 
For each $b>0$ let $R_{0}^{\prime}$ be the ring bounded by the disk $y_{1}^{2}+y_{2}^{2} \leqq b^{2}, y_{3}=0$ and by the surface of the cylinder $y_{1}^{2}+y_{2}^{2} \leqq(b+1)^{2},\left|y_{3}\right| \leqq 1$. Then let $R$ be the preimage of $R_{0}^{\prime}$ under the affine mapping

$$
y_{i}=a_{i} x_{i}, \quad i=1,2,3,
$$

and let $R_{n}^{\prime}$ be the image of $R$ under $n y(x / n)$. The $R_{n}^{\prime}$ are defined for large $n$ and (37) yields

$$
\bmod R_{n}^{\prime} \leqq K \bmod R \text {. }
$$

Moreover, since $y(x)$ is as in (40), each component of $\partial R_{n}^{\prime}$ converges uniformly to the corresponding component of $\partial R_{0}^{\prime}$ and Lemma 6 gives

$$
\bmod R_{0}^{\prime}=\lim _{n \rightarrow \infty} \bmod R_{n}^{\prime} \leqq K \bmod R .
$$

Now Lemma 7 and the above imply that

$$
\frac{s(R)^{3}}{m(R)^{2}} \leqq \Gamma(R) \leqq K^{2} \Gamma\left(R_{0}^{\prime}\right) \leqq K^{2} \frac{m\left(R_{0}^{\prime}\right)}{l\left(R_{0}^{\prime}\right)^{3}} .
$$

Then, since

$$
\begin{aligned}
l\left(R_{0}^{\prime}\right) & =1, \quad s\left(R_{0}^{\prime}\right)=a_{1} a_{2} s(R)=2 \pi b^{2}, \\
m\left(R_{0}^{\prime}\right) & =a_{1} a_{2} a_{3} m(R)=2 \pi(b+1)^{2},
\end{aligned}
$$

we obtain

$$
\frac{a_{3}^{2}}{a_{1} a_{2}} \leqq K^{2}\left(1+\frac{1}{b}\right)^{6} .
$$

Finally letting $b \rightarrow \infty$ yields $a_{3}^{2} \leqq K^{2} a_{1} a_{2}$ whence

$$
I(0)^{3}=a_{3}^{3} \leqq K^{2} a_{1} a_{2} a_{3}=K^{2} J(0)
$$

as desired.

Next fix $P^{\prime}$ in $D^{\prime}$ where $x(y)$ is differentiable. Again (39) follows from (36) when $J^{*}\left(P^{\prime}\right)=0$ and we may assume that $J^{*}\left(P^{\prime}\right)>0$. In this case $y(x)$ will have a differential at $P$ and, arguing as above, we may further assume that $P=P^{\prime}=0$ and that $y(x)$ is as in (40). Again $a_{3}^{2} \leqq K^{2} a_{1} a_{2}$ and we obtain

$$
I^{*}(0)^{3}=a_{1}^{-3} \leqq K^{4}\left(a_{1} a_{2} a_{3}\right)^{-1}=K^{4} J^{*}(0)
$$

completing the proof.

18. Analytic definition. We can now formulate an analytic definition for quasiconformal mappings in space.

Theorem 4. Let $y(x)$ be a topological mapping of a domain $D$. Then $y(x)$ satisfies the modulus condition 
$\bmod R^{\prime} \leqq K \bmod R$

for all bounded rings $R$ with $\bar{R} \subset D$ if and only if $y(x)$ is $A C L$ in $D$ with

$$
I(x)^{3} \leqq K^{2} J(x)
$$

a.e. in D. In particular $y(x)$ is $K$-quasiconformal if and only if $y(x)$ and $x(y)$ are $A C L$ with

$$
I(x)^{3} \leqq K^{2} J(x) \text { and } I^{*}(y)^{3} \leqq K^{2} J^{*}(y)
$$

a.e. in $D$ and $D^{\prime}$, respectively.

Proof. The necessity is an immediate consequence of Lemma 11 and the discussion which preceded it. For the sufficiency let $R$ be a bounded ring with $\bar{R} \subset D$, let $v$ be a continuously differentiable admissible function for $R^{\prime}$ and set $u(x)$ equal to $v(y(x))$ or to $1-v(y(x))$, depending on whether $B_{0}^{\prime}$ or $B_{1}^{\prime}$ is the boundary of the bounded component of $\mathfrak{C} R^{\prime}$. Then $u$ is admissible for $R$ and

a.e. in $R$. Hence

$$
|\nabla u(x)| \leqq|\nabla v(y(x))| I(x)
$$

$$
\Gamma(R) \leqq \int_{R}|\nabla u|^{3} d \omega \leqq K^{2} \int_{R}|\nabla v|^{3} J d \omega \leqq K^{2} \int_{R^{\prime}}|\nabla v|^{3} d \omega
$$

and taking the infimum over all such $v$ gives

$$
\Gamma(R) \leqq K^{2} \Gamma\left(R^{\prime}\right) \quad \text { or } \bmod R^{\prime} \leqq K \bmod R
$$

as desired.

The last part follows from applying the first part to both $y(x)$ and $x(y)$.

19. One sided modulus condition. An unfortunate feature of this analytic definition is that it involves both the mapping and its inverse. This is because our definition for quasiconformality is based on the two sided modulus condition (30). In the case of plane mappings, it is possible to define $K$-quasiconformal mappings using only a one sided condition. (See Theorem 3 of [10].) It is therefore natural to ask if this is also possible in space.

Theorem 5. Let $y(x)$ be a topological mapping of a domain $D$ and let $\bmod R^{\prime} \leqq K \bmod R$

for all bounded rings $R$ with $\bar{R} \subset D$. Then $y(x)$ is a $K^{2}$-quasiconformal mapping. The bound $K^{2}$ is best possible.

Proof. The discussion preceding Lemma 11 in $\$ 17$ shows that $y(x)$ and $x(y)$ are $A C L$ and a.e. differentiable. This last fact combined with Lemma 11 implies that

$$
I(x)^{3} \leqq K^{2} J(x) \text { and } I^{*}(y)^{3} \leqq K^{4} J^{*}(y)
$$


a.e. in $D$ and $D^{\prime}$, respectively. Hence $y(x)$ is $K^{2}$-quasiconformal by Theorem 4 .

It is easy to verify that the affine mapping

$$
y_{1}=x_{1}, \quad y_{2}=K^{2} x_{2}, \quad y_{3}=K^{2} x_{3}
$$

satisfies the above one sided modulus condition and is not $K_{1}$-quasiconformal for any $K_{1}<K^{2}$. Hence the bound $K^{2}$ cannot be improved.

20. Measurability. A topological mapping of a domain $D$ is said to be measurable if the image of every measurable set is itself measurable. We prove that quasiconformal mappings are measurable by establishing the following result.

TheOREM 6. Let $y(x)$ be a quasiconformal mapping of a domain $D$. Then $J(x)>0$ a.e. in D. Moreover if $E$ is a measurable set in $D$, then $E^{\prime}$ is measurable and

$$
m\left(E^{\prime}\right)=\int_{E} J d \omega .
$$

Proof. We begin by showing that $J(x)>0$ a.e. in $D$. For this assume that $y(x)$ is $K$-quasiconformal and let $E$ be the set where $J(x)=0$. Then $E$ is measurable and it is sufficient to show that $E$ has no points of density.

Fix $P$ in $D$. Next for $0<a<\rho(P, \partial D) / 2$ let $R$ be the spherical annulus $a<|x-P|<2 a$ and let $v$ be a continuously differentiable admissible function for $R^{\prime}$. Then $u(x)=v(y(x))$ is admissible for $R$ and $u$ is absolutely continuous on $X \cap R$ for almost all lines $X$ parallel to the $x_{1}$-axis. Hence

$$
\int_{X \cap_{R}}|\nabla u| d x_{1} \geqq 2
$$

for almost all $X$ which meet $C_{0}$ and hence $C_{1}$. Integrating and applying Fubini's theorem we obtain

$$
2 \pi a^{2} \leqq \int\left(\int_{X \cap_{R}}|\nabla u| d x_{1}\right) d x_{2} d x_{3} \leqq \int_{R}|\nabla u| d \omega \leqq \int_{R-E}|\nabla v| I d \omega,
$$

since $|\nabla u| \leqq|\nabla v| I$ a.e. in $R$ and $I=0$ in $E$ by Lemma 10 .

Hölder's inequality and Theorem 4 then yield

$$
\left(2 \pi a^{2}\right)^{3} \leqq K^{2} m(R-E)^{2} \int_{R}|\nabla v|^{3} J d \omega \leqq K^{2} m(R-E)^{2} \int_{R^{\prime}}|\nabla v|^{3} d \omega .
$$

Taking the infimum over all such $v$ gives

$$
\left(2 \pi a^{2}\right)^{3} \leqq K^{2} m(R-E)^{2} \Gamma\left(R^{\prime}\right),
$$

and hence

$$
\bmod R^{\prime} \leqq A K \frac{m(R-E)}{m(R)}
$$


where $A$ is an absolute constant. The modulus condition (30) implies that

$$
\frac{\log \dot{2}}{K}=\frac{1}{K} \bmod R \leqq \bmod R^{\prime}
$$

whence

$$
\liminf _{a \rightarrow 0} \frac{m(R-E)}{m(R)} \geqq \frac{\log 2}{A K^{2}}>0 .
$$

Thus $E$ has no points of density, $m(E)=0$ and $J(x)>0$ a.e. in $D$ as desired.

Now consider the nonnegative additive set function $\tau(E)=m\left(E^{\prime}\right)$ defined on the Borel sets $E \subset D$. Then $J(x)$ is the upper symmetric derivate of $\tau$. Let $E$ be the set where $J(x)=\infty$ and fix $P^{\prime}$ in $E^{\prime}$. Then either $x(y)$ does not have a differential at $P^{\prime}$ or $J^{*}\left(P^{\prime}\right)=0$, for otherwise $y(x)$ would have a differential at a point of $E$. Since $x(y)$ is quasiconformal, $m\left(E^{\prime}\right)=0$ and we can apply a form of the de la Vallée Poussin Decomposition Theorem to conclude that (41) holds for all bounded Borel sets $E \subset D$. (See p. 151 of [27].) From this it follows that the mapping $y(x)$ is measurable and that (41) holds for all measurable $E \subset D$, thus completing the proof for Theorem 6 .

21. Another criterion for quasiconformality. There are many ways of characterizing plane quasiconformal mappings. One of these states that a topological mapping $w(z)$ of a plane domain is $K$-quasiconformal if and only if $H(z)$ is bounded with $H(z) \leqq K$ a.e. (See Theorem 3 of $[6] ; H(z)$ is the plane analogue of the distortion function $H(x)$.)

It is natural to inquire about a space analogue for this characterization. We begin with the following result.

TheOREM 7. Let $y(x)$ be a topological mapping of a domain D. If $H(x)$ is bounded in $D$ with $H(x) \leqq K$ a.e., then $H^{*}(y)$ is bounded in $D^{\prime}$ with $H^{*}(y) \leqq K$ a.e.

Proof. We see from the discussion in $\$ 17$ that $y(x)$ is a.e. differentiable and $A C L$ in $D$. Then Lemma 10 , together with the fact that $H(x) \leqq K$ a.e. in $D$, yields

$$
I(x)^{3} \leqq K^{2} J(x)
$$

a.e. in $D$, and $y(x)$ is $K^{2}$-quasiconformal by Theorems 4 and 5 .

Now let $D-E$ be the set of points where $y(x)$ is differentiable with $H(x) \leqq K$ and $J(x)>0$. Theorem 6 implies that $E$ and its image are of zero measure and, since $H(x)=H^{*}(y)$ at corresponding points of these sets, we conclude that $H^{*}(y) \leqq K$ a.e. in $D^{\prime}$. Finally Lemma 8 and the fact that $x(y)$ is $K^{2}$-quasiconformal show that $H^{*}(y)$ is bounded and the proof is complete.

An immediate consequence of the above argument is that $y(x)$ is $K$-quasiconformal whenever $H(x)$ is bounded with $H(x) \leqq K$ a.e.

Now suppose that $y(x)$ is $K$-quasiconformal in $D$. Then $H(x)$ is bounded 
and $y(x)$ is differentiable with $J(x)>0$ in $D-E$ where $m(E)=0$. Fix $P$ in $D-E$. Arguing as in the proof for Lemma 11 we can assume that $P=P^{\prime}=0$ and that

$$
y_{i}=a_{i} x_{i}+o(|x|), \quad x_{i}=a_{i}^{-1} y_{i}+o(|y|), \quad i=1,2,3,
$$

where $0<a_{1} \leqq a_{2} \leqq a_{3}$. The two sided modulus condition (30) then implies that

$$
a_{3}^{2} \leqq K^{2} a_{1} a_{2}, \quad a_{1}^{-2} \leqq K^{2} a_{3}^{-1} a_{2}^{-1}
$$

and hence that $H(0) \leqq K^{4 / 3}$. Thus $H(x) \leqq K^{4 / 3}$ a.e. in $D$. The affine mapping

$$
y_{1}=x_{1}, \quad y_{2}=K^{4 / 3} x_{2}, \quad y_{3}=K^{2 / 3} x_{3}
$$

shows that the bound $K^{4 / 3}$ cannot be improved and we have thus established the following result.

Corollary 3. Let $y(x)$ be a topological mapping of a domain D. If $H(x)$ is bounded with $H(x) \leqq K$ a.e., then $y(x)$ is $K$-quasiconformal. If $y(x)$ is $K$-quasiconformal, then $H(x)$ is bounded with $H(x) \leqq K^{4 / 3}$ a.e. The bound $K^{4 / 3}$ is best possible.

In particular we are only able to give the following criterion for quasiconformality in terms of the function $H(x)$.

Corollary 4. A topological mapping $y(x)$ of a domain $D$ is quasiconformal if and only if $H(x)$ is bounded in $D$.

By slightly altering the definition for the distortion function $H(x)$, we could obtain a criterion for $K$-quasiconformality similar to that which holds in the plane. (See [28].) Or alternatively we might redefine the class of $K$ quasiconformal mappings as follows.

$A$ topological mapping $y(x)$ of a domain $D$ is said to be $K$-quasiconformal if $H(x)$ is bounded in $D$ with $H(x) \leqq K$ a.e. Theorem 7 shows that the inverse of such a mapping is again $K$-quasiconformal by this definition. Moreover any such mapping which is continuously differentiable with $J(x)>0$ is $K$ quasiconformal according to the classical definition. The example in (42) shows that this is not true for our class of $K$-quasiconformal mappings.

22. Exceptional sets. Corollary 4 can be extended as follows to yield information on the exceptional sets for quasiconformal mappings.

Theorem 8. Let $y(x)$ be a topological mapping of a domain $D$, let $H(x)$ be bounded a.e. in $D$ and let $H(x)<\infty$ in $D$ except possibly on a set of $\Sigma$-finite area. Then $y(x)$ is quasiconformal in $D$.

Proof. We first show that $y(x)$ is $A C L$. For this let $U$ be a sphere with $\bar{U} \subset D$ and let $X$ denote any line parallel to some fixed coordinate axis. We want to show that $y(x)$ is absolutely continuous on $U \cap X$ for almost all $X$. 
For each $r>0$ let $G$ denote the points of $U$ which lie within a distance $r$ of $X$. Next let $E_{b}$ denote the set where $H(x)$ is bounded and $E_{f}$ the set where $H(x)<\infty$. The above hypotheses combined with a theorem due to Gross (see p. 176 of [12]) imply that almost all lines $X$ have the following properties:

$$
T(X)=\limsup _{r \rightarrow 0} \frac{m\left(G^{\prime}\right)}{r^{2}}<\infty,
$$

$\left(U-E_{b}\right) \cap X$ has linear measure zero and $\left(U-E_{f}\right) \cap X$ is at most enumerable. Given any such line $X$ we can then follow almost verbatim the proof for Theorem 2 in [6] to show that $y(x)$ is absolutely continuous on $U \cap X$. Hence $y(x)$ is $A C L$ in $D$ as desired.

Next Lemma 10 and the fact that $H(x)$ is bounded a.e. imply the existence of a constant $K$ such that

$$
I(x)^{3} \leqq K J(x)
$$

a.e. in $D$. Hence $y(x)$ is $K$-quasiconformal by Theorems 4 and 5 and the proof is complete.

CoRollary 5. Let $y(x)$ be a topological mapping of a domain $D$, let $E$ be a set of $\Sigma$-finite area in $D$ and let $y(x)$ be K-quasiconformal in a neighborhood of each point of $D-E$. Then $y(x)$ is $K$-quasiconformal in $D$.

Proof. Lemma 8 implies that $H(x)$ is bounded in $D-E$ and hence $y(x)$ is quasiconformal in $D$ by Theorem 8. In particular $y(x)$ and $x(y)$ are $A C L$ in $D$ and $D^{\prime}$, respectively. Next Theorem 4 implies that

$$
I(x)^{3} \leqq K^{2} J(x) \text { and } I^{*}(y)^{3} \leqq K^{2} J^{*}(y)
$$

a.e. in $D-E$ and $D^{\prime}-E^{\prime}$, respectively. Since $m(E)=0$, Theorem 6 gives $m\left(E^{\prime}\right)=0$. Thus (43) holds a.e. in $D$ and $D^{\prime}$, respectively, and $y(x)$ is $K$-quasiconformal by Theorem 4 .

23. Extension to the boundary. We show here that a quasiconformal mapping of $|x|<1$ onto $|y|<1$ can be extended to give a topological mapping of $|x| \leqq 1$ onto $|y| \leqq 1$. We require first the following form of the inequality in Lemma 2.

LEMma 12. Let $u$ be continuous and $A C L$ in $x_{3}>0$ and for $r>0$ let $S$ be the hemispherical surface $|x|=r, x_{3}>0$. Then

$$
\int_{0}^{\infty}(\underset{S}{\operatorname{osc} u})^{3} \frac{d r}{r} \leqq 2 A \int_{x_{3}>0}|\nabla u|^{3} d \omega,
$$

where $A$ is as in Lemma 1.

Proof. For each $a>0$ let $v$ be the function which is equal to $u$ for $x_{3} \geqq a$ and is symmetric in the plane $x_{3}=a$, that is 


$$
v\left(x_{1}, x_{2}, x_{3}\right)=u\left(x_{1}, x_{2}, a+\left|x_{3}-a\right|\right) .
$$

Then $v$ is continuous and $A C L$ everywhere and Lemma 2 gives

$$
\int_{0}^{\infty}(\underset{|x|=r}{\operatorname{osc} v})^{3} \frac{d r}{r} \leqq A \int_{|x|<\infty}|\nabla v|^{3} d \omega \leqq 2 A \int_{x_{8}>0}|\nabla u|^{3} d \omega .
$$

It is easy to see that

$$
\underset{S}{\operatorname{osc}} u \leqq \underset{a \rightarrow 0}{\liminf } \underset{|x|=r}{\operatorname{osc}} v
$$

for each $r>0$, and applying Fatou's lemma to (45) yields (44).

TheORem 9. Let $y(x)$ be a quasiconformal mapping of $|x|<1$ onto $|y|<1$. Then $y(x)$ can be extended to a topological mapping of $|x| \leqq 1$ onto $|y| \leqq 1\left(^{5}\right)$.

Proof. We prove that $y(x)$ has a limit at each point of $|x|=1$. This means we can extend $y(x)$ so that it is continuous in $|x| \leqq 1$. Applying the same argument to $x(y)$ then shows that this extension is a topological mapping of $|x| \leqq 1$ onto $|y| \leqq 1$ as desired.

Suppose there exists a point $P$ of $|x|=1$ where $y(x)$ does not have a limit. By performing a preliminary inversion, rotation and translation we may assume that $y(x)$ is a $K$-quasiconformal mapping of $x_{3}>0$ onto $|y|<1$ which has no limit at the origin. Then we can find two sequences $\left\{P_{n}\right\}$ and $\left\{Q_{n}\right\}$ in $x_{3}>0$ which converge to the origin and for which $P_{n}^{\prime} \rightarrow P^{\prime}$ and $Q_{n}^{\prime} \rightarrow Q^{\prime}$ with $P^{\prime} \neq Q^{\prime}$. We can next pick points $P_{0}^{\prime}$ and $Q_{0}^{\prime}$ in $|y|<1$ such that the segments $P_{0}^{\prime} P^{\prime}$ and $Q_{0}^{\prime} Q^{\prime}$ lie at a distance $a>0$ from each other.

Now fix $0<b<\left|P_{0}\right|,\left|Q_{0}\right|$ and let $S$ denote the hemispherical surface $|x|=r, x_{3}>0$. If $0<r<b$, then $S^{\prime}$ will meet the segments $P_{0}^{\prime} P_{n}^{\prime}$ and $Q_{0}^{\prime} Q_{n}^{\prime}$ for all large $n$. This means that

$$
\underset{S}{\operatorname{osc}} y_{i} \geqq 3^{-1 / 2} a
$$

for some $i=i(r)$, where $y_{i}(x)$ is the $i$ th component of $y(x)$. Then, since $\left|\nabla y_{i}\right| \leqq I$ a.e., applying Lemma 12 to each $y_{i}$ and adding yields

$$
\infty=\int_{0}^{b}\left(3^{-1 / 2} a\right)^{3} \frac{d r}{r} \leqq 6 A \int_{x_{3}>0} I^{3} d \omega .
$$

But the fact that $y(x)$ maps $x_{3}>0 K$-quasiconformally onto $|y|<1$ implies that

$$
\int_{x_{3}>0} I^{3} d \omega \leqq K^{2} \int_{x_{3}>0} J d \omega<\infty
$$

(5) For a more general result, see [29]. 
and this contradicts (46). We conclude that $y(x)$ is continuous at $P$ and the proof is complete.

24. Boundary correspondence. Now let $y(x)$ be a $K$-quasiconformal mapping of $x_{3}>0$ onto $y_{3}>0$. By means of inversions we can apply Theorem 9 to conclude that $y(x)$ can be extended to a topological mapping of $x_{3} \geqq 0$ onto $y_{3} \geqq 0$. Reflection in the boundary planes $x_{3}=0$ and $y_{3}=0$ then yields a topological mapping of $|x|<\infty$ onto $|y|<\infty$ which is $K$-quasiconformal in a neighborhood of each point in $x_{3} \neq 0$. We can then apply Corollary 5 to conclude that the extended mapping $y(x)$ is $K$-quasiconformal in $|x|<\infty$.

Fix a point $P$ in the plane $x_{3}=0$ and let $\bar{H}(P)$ denote the distortion function, corresponding to $H(x)$, for the boundary correspondence between $x_{3}=0$ and $y_{3}=0$ induced by $y(x)$. Then

$$
\bar{H}(P) \leqq H(P)<e^{6 K}
$$

and we conclude that this plane mapping is also quasiconformal. The affine mapping in (42) shows, however, that this boundary correspondence need not be $K$-quasiconformal.

Summarizing we have established the following result.

THEOREM 10. Each quasiconformal mapping of $x_{3}>0$ onto $y_{3}>0$ can be extended to a topological mapping of $x_{3} \geqq 0$ onto $y_{3} \geqq 0$. The induced boundary correspondence is itself a plane quasiconformal mapping of $x_{3}=0$ onto $y_{3}=0$.

We note in particular that this boundary correspondence is absolutely continuous or measurable. An example due to Beurling and Ahlfors [2] shows this need not be true of the boundary correspondence induced by a quasiconformal mapping of a half plane onto a half plane.

25. General distortion theorem. We establish next a general distortion theorem for quasiconformal mappings in space. This result yields a space form of the Koebe Viertelsatz as well as an important theorem on normal families.

We begin with some notation. For $a>1$ we let $R_{G}=R_{G}(a)$ denote the ring bounded by the spherical surface $|x|=1$ and by the ray $a \leqq x_{1}<\infty$, $x_{2}=x_{3}=0$. Next for $b>0$ we let $R_{T}=R_{T}(b)$ denote the ring bounded by the segment $-1 \leqq x_{1} \leqq 0, x_{2}=x_{3}=0$ and by the ray $b \leqq x_{1}<\infty, x_{2}=x_{3}=0$. Finally we let

$$
\bmod R_{G}=\log \Phi(a), \quad \bmod R_{T}=\log \Psi(b) .
$$

From Lemma 6 of this paper and from Lemmas 6 and 8 of [9] it follows that $\Phi(a)$ is strictly increasing and continuous in $1<a<\infty$, that

$$
\lim _{a \rightarrow \infty} \frac{\Phi(a)}{a}=\lambda
$$

where $\lambda<12.4 \cdots$, and that 


$$
\Psi(b)=\Phi\left((b+1)^{1 / 2}\right)^{2} .
$$

Next for $1 \leqq K<\infty$ and $0<t<1$ we define

$$
\Theta_{K}(t)=\left(\Psi^{-1}\left(\Phi(1 / t)^{1 / K}\right)\right)^{-1},
$$

where $\Psi^{-1}$ is the inverse function for $\Psi$. Then $\Theta_{K}(t)$ is strictly increasing and continuous in $0<t<1$,

$$
\lim _{t \rightarrow 1} \Theta_{K}(t)=\infty
$$

and

$$
\lim _{t \rightarrow 0} \frac{\Theta_{K}(t)}{t^{1 / K}}=\lambda^{2-1 / K} .
$$

We now have the following distortion theorem.

THEOREM 11. Let $y(x)$ be a $K$-quasiconformal mapping of a domain $D$. If $\partial D$ is not empty, then $\partial D^{\prime}$ is not empty and

$$
\frac{\left|P^{\prime}-Q^{\prime}\right|}{\rho\left(P^{\prime}, \partial D^{\prime}\right)} \leqq \Theta_{K}\left(\frac{|P-Q|}{\rho(P, \partial D)}\right)
$$

for all $P$ and $Q$ in $D$ with $|P-Q|<\rho(P, \partial D)$.

Proof. A theorem due to Loewner [16] shows that $D^{\prime}$ is the whole space if and only if $D$ is. Thus $\partial D^{\prime}$ is empty if and only if $\partial D$ is.

Now fix $P$ and $Q$ in $D$ so that $|P-Q|<\rho(P, \partial D)$, let $|P-Q|<a<\rho(P, \partial D)$ and let $R$ be the ring bounded by the segment $P Q$ and by the surface $|x-P|$ $=a$. Then $\bar{R} \subset D$ and

$$
\bmod R=\log \Phi\left(\frac{a}{|P-Q|}\right) .
$$

Consider next the ring $R^{\prime}$. $C_{0}^{\prime}$ contains both $P^{\prime}$ and $Q^{\prime}$ while $B_{1}^{\prime}$ contains a point which lies at a distance $a^{\prime}<\rho\left(P^{\prime}, \partial D^{\prime}\right)$ from $P^{\prime}$. Hence Theorem 4 of [9] yields

$$
\bmod R^{\prime} \leqq \log \Psi\left(\frac{a^{\prime}}{\left|P^{\prime}-Q^{\prime}\right|}\right)
$$

Then, because $y(x)$ is $K$-quasiconformal,

$$
\bmod R^{\prime} \geqq \frac{1}{K} \bmod R
$$

and we obtain 


$$
\Psi\left(\frac{a^{\prime}}{\left|P^{\prime}-Q^{\prime}\right|}\right) \geqq \Phi\left(\frac{a}{|P-Q|}\right)^{1 / K}
$$

Thus

$$
\frac{\left|P^{\prime}-Q^{\prime}\right|}{\rho\left(P^{\prime}, \partial D^{\prime}\right)}<\frac{\left|P^{\prime}-Q^{\prime}\right|}{a^{\prime}} \leqq \Theta_{K}\left(\frac{|P-Q|}{a}\right),
$$

and letting $a \rightarrow \rho(P, \partial D)$ yields the desired inequality.

Corollary 6. Let $y(x)$ be a K-quasiconformal mapping of a domain $D$. Then for each compact set $E \subset D$ there exists a constant $A$ such that

$$
\left|P^{\prime}-Q^{\prime}\right| \leqq A|P-Q|^{1 / K}
$$

for all $P$ and $Q$ in $E$.

Proof. Suppose that $\partial D$ is not empty, let $E$ be any compact set in $D$ and let

$$
a=\rho(E, \partial D), \quad a^{\prime}=\rho\left(E^{\prime}, \partial D^{\prime}\right), \quad b^{\prime}=d\left(E^{\prime}\right), \quad 0<c<1 .
$$

Then by virtue of (49) we can find a constant $d$ such that

$$
\Theta_{K}(t) \leqq d t^{1 / K}
$$

for $0<t \leqq c$, and applying Theorem 11 yields

$$
\left|P^{\prime}-Q^{\prime}\right| \leqq A_{1}|P-Q|^{1 / K}, \quad A_{1}=\frac{a^{\prime}+b^{\prime}}{a^{1 / K}} d,
$$

for all $P$ and $Q$ in $E$ with $|P-Q| \leqq a c$. But clearly

$$
\left|P^{\prime}-Q^{\prime}\right| \leqq A_{2}|P-Q|^{1 / K}, \quad A_{2}=\frac{b^{\prime}}{(a c)^{1 / K}},
$$

for all $P$ and $Q$ in $E$ with $|P-Q| \geqq a c$ and we obtain the desired inequality by taking $A=\max \left(A_{1}, A_{2}\right)$.

Finally the case where $D$ is the whole space can be handled as above by first choosing $E$ and then replacing $D$ by any open sphere containing $E$.

26. Koebe Viertelsatz. Corollary 6 shows that a $K$-quasiconformal mapping satisfies a Hölder condition with exponent $1 / K$ at each point of the domain $D$. The following space form of the Koebe Viertelsatz gives us an asymptotic estimate for the coefficient in this Hölder condition. (Compare with Theorem $4^{\prime}$ of $[23]$.)

ThEOREM 12. Let $y(x)$ be a $K$-quasiconformal mapping of a domain $D$ and let $\partial D$ be nonempty. Then

$$
\limsup _{r \rightarrow 0} \frac{L(P, r)}{r^{1 / K}} \leqq \lambda^{2-1 / K} \frac{\rho\left(P^{\prime}, \partial D^{\prime}\right)}{\rho(P, \partial D)^{1 / K}}
$$


and

$$
\limsup _{r \rightarrow 0} \frac{l(P, r)}{r^{1 / K}} \leqq \lambda \frac{\rho\left(P^{\prime}, \partial D^{\prime}\right)}{\rho(P, \partial D)^{1 / K}}
$$

for each $P$ in $D$, where $\lambda$ is as in $(47)\left({ }^{6}\right)$.

Proof. For the first part fix $0<r<\rho(P, \partial D)$. Then Theorem 11 implies that

$$
\frac{L(P, r)}{r^{1 / K}} \leqq \frac{\rho\left(P^{\prime}, \partial D^{\prime}\right)}{\rho(P, \partial D)^{1 / K}} \frac{\Theta_{K}(t)}{t^{1 / K}}, \quad t=\frac{r}{\rho(P, \partial D)},
$$

and we obtain (51) from (49) by letting $r \rightarrow 0$.

For the second part fix $0<r<a<\rho(P, \partial D)$ and let $R$ be the ring $r<|x-P|$ $<a$. Then

$$
\bmod R=\log \frac{a}{r}
$$

Consider next the ring $R^{\prime} . C_{0}^{\prime}$ contains the sphere $\left|y-P^{\prime}\right| \leqq r^{\prime}=l(P, r)$ while $B_{1}^{\prime}$ contains a point which lies at distance $a^{\prime}<\rho\left(P^{\prime}, \partial D^{\prime}\right)$ from $P^{\prime}$. Hence arguing exactly as in the proof of Theorem 4 in [9] we see that

$$
\bmod R^{\prime} \leqq \log \Phi\left(\frac{a^{\prime}}{r^{\prime}}\right) \leqq \log \lambda \frac{a^{\prime}}{r^{\prime}}
$$

The modulus condition (50) yields

$$
\frac{l(P, r)}{r^{1 / K}} \leqq \lambda \frac{\rho\left(P^{\prime}, \partial D^{\prime}\right)}{a^{1 / K}}
$$

and we obtain (52) from first letting $r \rightarrow 0$ and then $a \rightarrow \rho(P, \partial D)$.

Theorem 12 yields a lower bound for the constant $\lambda$. For let $P$ be the point $(1,0,0), D$ the sphere $|x-P|<1$ and $D^{\prime}$ the half space $y_{1}>2$. Then the inversion

$$
y(x)=\frac{4 x}{|x|^{2}}
$$

maps $D$ 1-quasiconformally onto $D^{\prime}$ and Theorem 12 gives

$$
4=I(P) \leqq \lambda \frac{\rho\left(P^{\prime}, \partial D^{\prime}\right)}{\rho(P, \partial D)}=2 \lambda \text {. }
$$

Hence $\lambda \geqq 2$.

27. Normal families. Let $D$ and $D^{\prime}$ be a pair of domains with nonempty boundaries. We show here that the $K$-quasiconformal mappings of $D$ onto $D^{\prime}$,

( $)$ The plane analogue for Theorem 12 has $\lambda=4$. In this case (51) and (52) each yield the Koebe Viertelsatz when $K=1$. 
which preserve the correspondence between a pair of points, form a closed normal family.

We begin with the following result.

Theorem 13. Let $D$ and $D^{\prime}$ be domains, let $\partial D$ be nonempty and let $\left\{y_{n}(x)\right\}$ be a sequence of $K$-quasiconformal mappings of $D$ onto $D^{\prime}$. Then either

$$
\lim _{n \rightarrow \infty}\left|y_{n}(x)\right|=\infty
$$

for all $x$ in $D$ or there exists a subsequence which converges uniformly on each compact set in $D$ to a function $y(x)$. This limit function is either a constant vector in $\partial D^{\prime}$ or a $K$-quasiconformal mapping of $D$ onto $D^{\prime}$.

Proof. Let $G$ be the set of $x$ in $D$ for which

$$
\limsup _{n \rightarrow \infty}\left|y_{n}(x)\right|<\infty \text {. }
$$

We show first that $G$ is either empty or equal to the whole of $D$. For this fix $P$ in $G$ and pick $Q$ so that $|P-Q|<\rho(P, \partial D) / 2$. Then Theorem 11 yields

$$
\left|y_{n}(Q)\right| \leqq\left|y_{n}(P)\right|+\rho\left(y_{n}(P), \partial D^{\prime}\right) \Theta_{K}\left(\frac{1}{2}\right)
$$

and, since the quantities on the right are bounded, we conclude that $Q$ is in $G$. Hence $G$ is open. Similarly if $Q$ is in $D-G$ and if $P$ is chosen so that $|P-Q|<\rho(Q, \partial D) / 3$, then $|P-Q|<\rho(P, \partial D) / 2$ and (54) implies that $P$ is in $D-G$. Hence $D-G$ is also open and, since $D$ is connected, we obtain the desired conclusion.

From the above we now see that either (53) holds for all $x$ in $D$ or there exists a subsequence $\left\{y_{n_{k}}(x)\right\}$ such that

$$
\limsup _{k \rightarrow \infty}\left|y_{n_{k}}(x)\right|<\infty
$$

for all $x$ in $D$. Theorem 11 then shows that the $y_{n_{k}}(x)$ are equicontinuous in $D$ and we can apply Ascoli's theorem to find a second subsequence which converges uniformly on each compact set in $D$ to a function $y(x)$. To complete the proof we must show that $y(x)$ is either a constant vector in $\partial D^{\prime}$ or a $K$-quasiconformal mapping of $D$ onto $D^{\prime}$.

By relabeling we may assume that the original sequence $\left\{y_{n}(x)\right\}$ converges to $y(x)$. Now fix a point $P^{\prime}$ in $\partial D^{\prime}$ and let $G$ be the set of $x$ in $D$ for which $y(x)=P^{\prime}$. Since $y(x)$ is continuous, $G$ is closed in $D$. On the other hand if $P$ is in $G$ and if $|P-Q|<\rho(P, \partial D) / 2$, then Theorem 11 yields

$$
\left|y(Q)-P^{\prime}\right|=\lim _{n \rightarrow \infty}\left|y_{n}(Q)-y_{n}(P)\right| \leqq \liminf _{n \rightarrow \infty} \rho\left(y_{n}(P), \partial D^{\prime}\right) \Theta_{K}\left(\frac{1}{2}\right)=0 \text {. }
$$

Hence $Q$ is in $G$ and $G$ is open. We conclude that either $y(x)$ is a constant 
vector in $\partial D^{\prime}$ or that $y(P)$ is in $D^{\prime}$ for all $P$ in $D$.

We must prove that in this last case $y(x)$ is a $K$-quasiconformal mapping of $D$ onto $D^{\prime}$. We begin by showing that

$$
\lim _{n \rightarrow \infty} x_{n}(y(P))=P
$$

for all $P$ in $D$, where $x_{n}(y)$ is the inverse of $y_{n}(x)$. For this fix $P$ in $D$. Then $y(P)$ is in $D^{\prime}$ and Theorem 11 applied to $x_{n}(y)$ gives

$$
\left|x_{n}(y(P))-P\right| \leqq \rho(P, \partial D) \Theta_{K}\left(\frac{\left|y(P)-y_{n}(P)\right|}{\rho\left(y_{n}(P), \partial D^{\prime}\right)}\right)
$$

for large $n$, and letting $n \rightarrow \infty$ yields (55).

Next all of the above arguments can be applied to the sequence $\left\{x_{n}(y)\right\}$. In particular the conclusion of the first paragraph combined with (55) implies that

$$
\limsup _{n \rightarrow \infty}\left|x_{n}(y)\right|<\infty
$$

for all $y$ in $D^{\prime}$. Hence the $x_{n}(y)$ are equicontinuous in $D^{\prime}$ and, by Ascoli's theorem, a subsequence converges uniformly on each compact subset of $D^{\prime}$ to a continuous function $x(y)$. Relabeling allows us to replace this subsequence by the sequence $\left\{x_{n}(y)\right\}$.

Then (55) implies that $x(y(P))=P$ for all $P$ in $D$. It then follows that $x\left(P^{\prime}\right)$ is in $D$ for all $P^{\prime}$ in $D^{\prime}$ and arguing as above we conclude that $y\left(x\left(P^{\prime}\right)\right)$ $=P^{\prime}$ for all $P^{\prime}$ in $D^{\prime}$. Thus $y(x)$ is a topological mapping of $D$ onto $D^{\prime}$. Theorem 3 implies that $y(x)$ is $K$-quasiconformal and the proof for Theorem 13 is complete.

The following is now an immediate consequence of Theorem 13.

Corollary 7. Let $D$ and $D^{\prime}$ be domains, let $\partial D$ be nonempty and let $P$ and $P^{\prime}$ be fixed points in $D$ and $D^{\prime}$, respectively. Then the $K$-quasiconformal mappings of $D$ onto $D^{\prime}$ which map $P$ onto $P^{\prime}$ form a closed normal family.

28. Special distortion theorem. When $D$ and $D^{\prime}$ are spheres we get the following strengthened version of Corollary 6 . It is the space analogue of a theorem due to Ahlfors [1] and Mori [18].

TheOREM 14. Let $y(x)$ be a K-quasiconformal mapping of $|x|<1$ onto $|y|<1$ and let $y(0)=0$. There exists an absolute constant $A$ such that

$$
\left|P^{\prime}-Q^{\prime}\right| \leqq A|P-Q|^{1 / K}
$$

for all $P$ and $Q$ in $|x|<1$.

Proof. We can extend $y(x)$ to a $K$-quasiconformal mapping of the whole space onto itself by means of Theorems 8 and 9 and reflection. Next pick $P$ 
and $Q$ in $|x|<1$ and let $R$ be the spherical annulus $|P-Q|<|x-P|<1+|P|$. Then clearly

$$
\bmod R=\log \frac{1+|P|}{|P-Q|} \geqq \log \frac{1}{|P-Q|} .
$$

For an estimate for $\bmod R^{\prime}$ we observe that $P^{\prime}$ and $Q^{\prime}$ are in $C_{0}^{\prime}$ and that $C_{1}^{\prime}$ meets $|y|=1$. Thus $C_{0}^{\prime}$ and $C_{1}^{\prime}$ contain points which lie at distances $\left|P^{\prime}-Q^{\prime}\right|$ and $1+\left|P^{\prime}\right|$ from $P^{\prime}$ and we can apply Theorem 4 of [9] to obtain

$$
\bmod R^{\prime} \leqq \log \Psi\left(\frac{1+\left|P^{\prime}\right|}{\left|P^{\prime}-Q^{\prime}\right|}\right) \leqq \log \frac{4 \lambda^{2}}{\left|P^{\prime}-Q^{\prime}\right|} .
$$

Since $y(x)$ is $K$-quasiconformal,

$$
\bmod R^{\prime} \geqq \frac{1}{K} \bmod R
$$

and this, together with the above inequalities, yields (56) with $A=4 \lambda^{2}<620$.

29. The 1-quasiconformal mappings. If $y(x)$ is a topological mapping which is the restriction of a Moebius transformation to a domain $D$, then $y(x)$ is a 1-quasiconformal mapping of $D$. The following result shows that the converse is true, that is every 1-quasiconformal mapping of $D$ is the restriction of a Moebius transformation to $D$.

TheOREM 15. Let $y(x)$ be a topological mapping of a domain $D$ and let $\bmod R^{\prime} \leqq \bmod R$

for all bounded rings $R$ with $\bar{R} \subset D$. Then $y(x)$ is the restriction of a Moebius transformation to the domain $D$.

Proof. Theorem 5 implies that $y(x)$ is 1 -quasiconformal. Next Corollary 6 implies that $I(x)$ is bounded away from 0 and $\infty$ on each compact set in $D$ while Theorem 4 and Corollary 3 imply that $I(x)^{3} \leqq J(x)$ and $H(x)=1$ a.e. in $D$. The remainder of the proof is devoted to showing that $y(x)$ satisfies the hypotheses of the following classical result due to Liouville. (See, for example, [22].)

Liouville's TheOREM. Let $y(x)$ be a topological mapping of a domain $D$, let $y(x)$ have continuous fourth order partial derivatives and let

$$
J(x)>0 \text { and } H(x)=1
$$

everywhere in $D$. Then $y(x)$ is the restriction of a Moebius transformation to $D$.

It is clear we need only show that $y(x)$ is $C^{4}$ in $D$, for ther (57) will follow for all $x$ in $D$ by continuity. Moreover for the $C^{4}$ differentiability it is sufficient to show there exists an absolute constant $a>0$ with the following property: 
for each $P$ in $D$ the function $\left|y(x)-P^{\prime}\right|$ is real analytic in the punctured sphere $0<|x-P|<a \rho(P, \partial D)$.

Let $\Theta_{1}(t)$ be the distortion function given in (48) with $K=1$ and pick $0<a<1$ so $\Theta_{1}(a)<1$. Now fix $P$ in $D$ and let $R^{\prime}$ be the ring $c<\left|y-P^{\prime}\right|<d$ where $0<c<d=L(P, b)$ and $b=a \rho(P, \partial D)$. Theorem 11 yields

$$
L(P, b) \leqq \rho\left(P^{\prime}, \partial D^{\prime}\right) \Theta_{1}(a)<\rho\left(P^{\prime}, \partial D^{\prime}\right)
$$

and hence $\bar{R}^{\prime} \subset D^{\prime}$.

Next let $v$ be the extremal function for $R^{\prime}$ and set $u(x)=v(y(x))$. Then we see that

$$
v(y)=\frac{\log \left|\frac{y-P^{\prime}}{c}\right|}{\log \frac{d}{c}},
$$

and that $u$ is admissible for $R$ with $|\nabla u| \leqq|\nabla v| I$ a.e. in $R$. Hence

$$
\Gamma(R) \leqq \int_{R}|\nabla u|^{3} d \omega \leqq \int_{R}|\nabla v|^{3} J d \omega \leqq \int_{R^{\prime}}|\nabla v|^{3} d \omega=\Gamma\left(R^{\prime}\right)=\Gamma(R)
$$

and $u$ is the extremal function for $R$.

Corollary 6 implies we can find a positive constant $M$ such that $I(x) \leqq M$ in $R$ and $I^{*}(y) \leqq M$ in $R^{\prime}$. This implies that

$$
\frac{1}{M d}\left(\log \frac{d}{c}\right)^{-1} \leqq \limsup _{h \rightarrow 0} \frac{|u(x+h)-u(x)|}{|h|} \leqq \frac{M}{c}\left(\log \frac{d}{c}\right)^{-1}
$$

everywhere in $R$. Thus $u$ satisfies the hypotheses of Theorem 2 and is real analytic in $R$. This means that

$$
\left|y(x)-P^{\prime}\right|=c\left(\frac{d}{c}\right)^{u(x)}
$$

is real analytic in $R$ and, letting $c \rightarrow 0$, we conclude that $\left|y(x)-P^{\prime}\right|$ is real analytic in $0<|x-P|<a \rho(P, \partial D)$ as desired.

30. Liouville's theorem. For some time it has been an open question as to what a priori differentiability hypotheses are necessary in Liouville's theorem on the conformal mappings in space. The theorem stated in $\$ 29$ asks that the mapping have continuous fourth order partial derivatives.

The following general result shows that no such differentiability hypotheses are necessary. It is the space form of a theorem due to Menchoff [17]. (See also Corollary 3 in [6].)

TheOREM 16. Let $y(x)$ be a topological mapping of a domain $D$, let $H(x)=1$ 
a.e. in $D$ and let $H(x)<\infty$ in $D$ except possibly on a set of $\Sigma$-finite area. Then $y(x)$ is the restriction of a Moebius transformation to $D\left({ }^{7}\right)$.

Proof. Theorem 8 implies that $y(x)$ is quasiconformal in $D$. Hence $H(x)$ is bounded in $D$ and Corollary 3 implies that $y(x)$ is 1-quasiconformal there. The desired conclusion then follows from Theorem 15.

In the above theorem nothing is said about $H(x)$ in an exceptional set $E$ which is required to be of $\Sigma$-finite area. The following example shows why this requirement on $E$ is necessary.

Let $X_{i}$ denote the $i$ th coordinate axis, let $F$ be any nonenumerable closed set in $X_{1}$ and let $f\left(x_{1}\right)$ be a continuous nondecreasing singular function which is constant in each interval of $X_{1}-F$. Then the mapping

$$
y_{1}=x_{1}+f\left(x_{1}\right), \quad y_{2}=x_{2}, \quad y_{3}=x_{3}
$$

is a topological mapping of the space onto itself for which $H(x)=1$ in $\mathfrak{e} E$ where $E=F \times X_{2} \times X_{3}$. But $y(x)$ is clearly not a Moebius transformation.

31. Coefficient of quasiconformality. Theorem 15 shows that spheres and half spaces are the only domains which can be mapped 1-quasiconformally onto the unit sphere $|y|<1$. This contrasts with the situation in the plane where we have the Riemann mapping theorem. It is therefore natural to ask if there are other domains which can be mapped $K$-quasiconformally onto $|y|<1$ for $K$ arbitrarily close to 1 .

We can reformulate this question as follows. For each domain $D$ let $K(D)$ denote the infimum of the numbers $K$ for which there exists a $K$-quasiconformal mapping of $D$ onto $|y|<1$; if no such mappings exist set $K(D)=\infty$. We call $K(D)$ the coefficient of quasiconformality for $D$. It measures how far from conformal a mapping must be in order to map $D$ onto $|y|<1$.

The above question then asks us to identify the domains $D$ for which $K(D)=1$.

Theorem 17. Let $D$ be a domain and let $K(D)<\infty$. For each $P$ in $D$ there exists a $K(D)$-quasiconformal mapping of $D$ onto $|y|<1$ with $y(P)=0$.

Proof. Let $\left\{K_{n}\right\}$ be a decreasing sequence which converges to $K(D)$. For each $n$ we can find a $K_{n}$-quasiconformal mapping $y_{n}(x)$ of $D$ onto $|y|<1$ and, by performing a preliminary inversion, we can arrange that $y_{n}(P)=0$. By Theorem 13 a subsequence converges uniformly on each compact subset of $D$ to a topological mapping $y(x)$ of $D$ onto $|y|<1$. Hence $y(P)=0$. Now let $R$ be any bounded ring with $\bar{R} \subset D$. Then Theorem 3 implies that

$$
\frac{1}{K_{n}} \bmod R \leqq \bmod R^{\prime} \leqq K_{n} \bmod R
$$

(7) While this manuscript was in preparation, a paper by Rešetnyak [26] appeared in which a similar theorem is considered. 
for all $n$. Letting $n \rightarrow \infty$ yields (58) with $K(D)$ in place of $K_{n}$ and hence $y(x)$ is $K(D)$-quasiconformal as desired.

We see from Theorems 15 and 17 that $K(D)=1$ if and only if $D$ is a sphere or a half space.

32. A compactness characterization for quasiconformality. Let $F$ be a family of topological mappings $y=y(x)$ of the whole space. We say that $F$ is complete with respect to similarity mappings if, given any $y$ in $F$, the composed mapping $S y T$ is also in $F$ for each pair of similarity mappings $S$ and $T$.

Next let 0 denote the origin and $0_{1}$ the point $(1,0,0)$. We say that a mapping $y$ in $F$ is normalized if $y(0)=0$ and $y\left(0_{1}\right)=0_{1}$. Then the family $F$ is said to satisfy the compactness condition (A) if every infinite set of normalized mappings in $F$ contains a subsequence which converges uniformly on compact sets to a topological mapping.

We have the following interpretation for this compactness condition.

THEOREM 18. Let $F$ be a family of topological mappings of the space which is complete with respect to similarity mappings. Then $F$ satisfies the compactness condition (A) if and only if each mapping in $F$ is $K$-quasiconformal for some fixed $K$.

Proof. Suppose first that the mappings in $F$ are $K$-quasiconformal. Corollary 7 implies that the $K$-quasiconformal mappings of the space which keep 0 and $0_{1}$ fixed constitute a closed normal family. Hence each sequence of normalized mappings in $F$ contains a subsequence which converges uniformly on each compact set to a $K$-quasiconformal mapping of the space, and $F$ clearly satisfies the compactness condition (A).

For the converse let

$$
K=\sup \left(\max _{|x|=1}|y(x)|\right),
$$

where the supremum is taken over all normalized mappings $y$ in $F$. Then there exists a sequence of normalized mappings $y_{n}$ in $F$ for which

$$
K=\lim _{n \rightarrow \infty}\left(\max _{|x|=1}\left|y_{n}(x)\right|\right)
$$

and, since a subsequence tends uniformly on $|x|=1$ to a topological mapping, $1 \leqq K<\infty$.

Now let $y(x)$ be any mapping in $F$, fix $P$ and $r>0$ and choose $Q$ on $|x-P|=r$ so that $\left|P^{\prime}-Q^{\prime}\right|=l(P, r)$. Next let $T$ and $S$ be the similarity mappings which map $|x|=1$ onto $|x-P|=r$ and $\left|y-P^{\prime}\right|=l(P, r)$ onto $|y|=1$, respectively, so that

$$
T(0)=P \quad T\left(0_{1}\right)=Q, \quad S\left(P^{\prime}\right)=0, \quad S\left(Q^{\prime}\right)=0_{1} .
$$


Then $S y T$ is a normalized mapping in $F$ and we thus obtain

$$
L(P, r)=l(P, r) \max _{|x|=1}|S(y(T(x)))| \leqq K l(P, r) .
$$

Letting $r \rightarrow 0$ yields $H(P) \leqq K$ and we conclude that $y(x)$ is $K$-quasiconformal from Corollary 3.

If we let $S$ and $T$ denote arbitrary similarity mappings, then Theorem 18 yields the following characterization for quasiconformal mappings of the space.

Corollary 8. A topological mapping $y(x)$ of the space is quasiconformal if and only if the family of all mappings SyT satisfies the compactness condition (A).

Corollary 8 is the space form of a result due to Beurling and Ahlfors (Corollary of [2]). Viewed in this light it is clear that one should interpret the topological mappings of the real line which satisfy a $\rho$-condition as 1 -dimensional quasiconformal mappings.

\section{REFERENCES}

1. L. V. Ahlfors, On quasiconformal mappings, J. Analyse Math. 3 (1954), 1-58.

2. A. Beurling and L. V. Ahlfors, The boundary correspondence under quasiconformal mappings, Acta Math. 96 (1956), 125-142.

3. E. D. Callender, Hölder continuity of $N$-dimensional quasi-conformal mappings, Pacific J. Math. 10 (1960), 499-515.

4. J. A. Clarkson, Uniformly convex spaces, Trans. Amer. Math. Soc. 40 (1936), 396-414.

5. H. Federer, Curvature measures, Trans. Amer. Math. Soc. 93 (1959), 418-491.

6. F. W. Gehring, The definitions and exceptional sets for quasiconformal mappings, Ann. Acad. Sci. Fenn. Ser. A 281 (1960), 1-28.

7. - Rings and quasiconformal mappings in space, Proc. Nat. Acad. Sci. U.S.A. 47 (1961), 98-105.

8. - A remark on the moduli of rings, Comment. Math. Helv. 36 (1961), 42-46.

9. - Symmetrization of rings in space, Trans. Amer. Math. Soc. 101 (1961), 499-519.

10. F. W. Gehring and J. Väisälä, On the geometric definition for quasiconformal mappings, Comment. Math. Helv. 36 (1961), 19-32.

11. E. de Giorgi, Sulla differenziabilità e l'analiticità delle estremali degli integrali multipli regolari, Mem. Accad. Sci. Torino Cl. Sci. Fis. Mat. Nat. 3 (1957), 25-43.

12. W. Gross, Über das Flächenmass von Punktmengen, Monatsh. Math. 29 (1918), 145-176.

13. G. H. Hardy, J. E. Littlewood and G. P6lya, Inequalities, Cambridge Univ. Press, 1934.

14. E. Hopf, Über den funktionalen, inbesondere den analytischen Charakter der Lösungen elliptischer Differentialgleichungen zweiter Ordnung, Math. Z. 34 (1932), 194-233.

15. H. Lebesgue, Sur le probleme de Dirichlet, Rend. Circ. Mat. Palermo 24 (1907), 371-402.

16. C. Loewner, On the conformal capacity in space, J. Math. Mech. 8 (1959), 411-414.

17. D. Menchoff, Sur une généralisation d'un théorème de M. H. Bohr, Mat. Sb. 44 (1937), 339-354.

18. A. Mori, On an absolute constant in the theory of quasi-conformal mappings, J. Math. Soc. Japan 8 (1956), 156-166. 
19. - On quasi-conformality and pseudo-analyticity, Trans. Amer. Math. Soc. 84 (1957), 56-77.

20. C. B. Morrey, Second order elliptic systems of differential equations, Annals of Mathematics Studies No. 33, pp. 101-159, Princeton Univ. Press, Princeton, N. J. 1954.

21. J. Moser, $A$ new proof of de Giorgi's theorem concerning the regularity problem for elliptic differential equations, Comm. Pure Appl. Math. 13 (1960), 457-468.

22. R. Nevanlinna, On differentiable mappings, Analytic Functions, pp. 3-9, Princeton Univ. Press, Princeton, N. J., 1960.

23. A. Pfluger, Quasikonforme Abbildungen und logarithmische Kapazität, Ann. Inst. Fourier Grenoble 2 (1950), 69-80.

24. T. Rad6 and P. V. Reichelderfer, Continuous transformations in analysis, SpringerVerlag, Berlin, 1955.

25. Yu. Rešetnyak, A sufficient condition for Hölder continuity of a mapping, Soviet Math. Dokl. 1 (1960), 76-78.

26. - On conformal mappings of a space, Soviet Math. Dokl. 1 (1960), 153-155.

27. S. Saks, Theory of the integral, Warsaw, 1937.

28. J. Väisälä, On quasiconformal mappings in space, Ann. Acad. Sci. Fenn. Ser. A 298 (1961), 1-36. $1-7$.

29. - On quasiconformal mappings of a ball, Ann. Acad. Sci. Fenn. Ser. A 304 (1961),

UNiversity OF Michigan, Ann Arbor, Michigan

EIDGENÖSSISCHE TECHNISChe Hochschule, ZÜRICH, SWITZERLAND 